\title{
A classifier for spurious astrometric solutions in Gaia eDR3
}

\author{
Jan Rybizki $^{1 \dagger}$, Gregory M. Green ${ }^{1 \star \dagger}$, Hans-Walter Rix ${ }^{1}$, Kareem El-Badry ${ }^{1}$, \\ Markus Demleitner ${ }^{2}$, Eleonora Zari ${ }^{1}$, Andrzej Udalski ${ }^{3}$, Richard L. Smart ${ }^{4}$, \\ and Andrew Gould ${ }^{1,5}$ \\ ${ }^{\dagger}$ These authors contributed equally \\ ${ }^{1}$ Max Planck Institute for Astronomy, Königstuhl 17, D-69117 Heidelberg, Germany \\ ${ }^{2}$ Astronomisches Rechen-Institut, Zentrum für Astronomie der Universität Heidelberg, Mönchhofstrasse 12-14, D-69120 Heidelberg, Germany \\ ${ }^{3}$ Astronomical Observatory, University of Warsaw, Al. Ujazdowskie 4, 00-478 Warszawa, Poland \\ ${ }^{4}$ INAF - Osservatorio Astrofisico di Torino, via Osservatorio 20, 10025 Pino Torinese (TO), Italy \\ ${ }^{5}$ Department of Astronomy, Ohio State University, 4055 McPherson Laboratory, 140 West 18th Avenue, Columbus, Ohio 43210, USA
}

Accepted 06-Dec-2021. Received 10-Sep-2021

\begin{abstract}
The Gaia early Data Release 3 has delivered exquisite astrometric data for 1.47 billion sources, which is revolutionizing many fields in astronomy. For a small fraction of these sources, the astrometric solutions are poor, and the reported values and uncertainties may not apply. Before any analysis, it is important to recognize and excise these spurious results - this is commonly done by means of quality flags in the Gaia catalog. Here, we devise a means of separating "good" from "bad" astrometric solutions that is an order of magnitude cleaner than any single flag: $99.3 \%$ pure and $97.3 \%$ complete, as validated on our test data. We devise an extensive sample of manifestly bad astrometric solutions, with parallax that is negative at $\geq 4.5 \sigma$; and a corresponding sample of presumably good solutions, including sources in HEALPix pixels on the sky that do not contain such negative parallaxes, and sources that fall on the main sequence in a color-absolute magnitude diagram. We then train a neural network that uses 17 pertinent Gaia catalog entries and information about nearby sources to discriminate between these two samples, captured in a single "astrometric fidelity" parameter. A diverse set of verification tests shows that our approach works very cleanly, including for sources with positive parallaxes. The main limitations of our approach are in the very low-SNR and the crowded regime. Our astrometric fidelities for all of eDR3 can be queried via the Virtual Observatory, our code and data are public.
\end{abstract}

Key words: Galaxy: stellar content, Galaxy: kinematics and dynamics, software: public release, space vehicles: instruments, virtual observatory tools

\section{INTRODUCTION}

Parallax measurements contain information about the distance to astrophysical objects, and are critical to anchoring the cosmic distance ladder. At the same time, kinematic measurements - proper motions and radial velocities - provide phase-space information that is key to understanding Milky Way dynamics and external galaxies. The 1.47 billion astrometric measurements reported in Gaia Early Data Release 3 (Gaia Collaboration et al. 2021a, "eDR3") constitute the largest astrometric dataset ever produced.

While this astrometric catalog is of extremely high quality (Lindegren et al. 2021a), a significant fraction of astrometric solutions are spurious (Fabricius et al. 2021). Spurious astrometric solutions are a distinct issue from negative parallaxes, which are

\footnotetext{
^ E-mail: green@mpia.de
}

an expected outcome of the normally distributed parallax measurement (Bailer-Jones 2015; Luri et al. 2018). Spurious solutions result from specific failure modes (Fabricius et al. 2021), and as an ensemble, have true parallaxes that are incompatible with the reported catalog values (and their uncertainties). This can be a particular concern when looking at sparsely populated portions of the colormagnitude diagram, or at extreme objects, such as the nearest or fastest-moving stars (i.e., those with the largest parallaxes or proper motions, respectively). For example, naively selecting all objects with measured parallaxes greater than 10 mas (corresponding to a distance of less than $100 \mathrm{pc}$ ) yields a catalog with an estimated $50 \%$ of spurious parallax measurements. Gaia eDR3 provides a number of astrometric quality parameters that can be used to exclude such spurious solutions. The "Gaia Catalogue of Nearby Stars" (Gaia Collaboration et al. 2021b, "GCNS") uses a combination of these parameters to filter out spurious sources, obtaining a highly complete and pure subset of Gaia eDR3 sources lying within $100 \mathrm{pc}$. 
In this paper, we use a similar approach to extend this work to the entire Gaia eDR3 catalog.

Gaia eDR3 provides 1.47 billion astrometric measurements containing of a two-dimensional position on the sky, a twodimensional proper motion and a parallax (in addition, a 7.2M subset also has radial velocity measurements). There are many possible sources of excess noise in these astrometric measurements. Some error modes, such as unmodeled acceleration caused by an unresolved binary companion typically introduce small residuals into the astrometric solution, which will usually be accounted for in the parallax uncertainty estimate (Lindegren et al. 2021a). However, other error modes, such as incorrect epoch cross-matches with background or spurious sources and also close source pairs, which might be partially resolved (Fabricius et al. 2021), can introduce very large residuals, scattered around the true parallax (Gaia Collaboration et al. 2021b), which are unaccounted for in the reported parallax uncertainty. Spurious astrometric solutions mainly happen in very dense parts of the sky (Fabricius et al. 2021). It is this latter class of "catastrophic" errors in the astrometric solutions (leading to errors in excess of the stated uncertainties) that we will attempt to detect. We will ignore the fact that the reported parallax uncertainties in eDR3 are slightly underestimated (Zinn 2021) and can be significantly underestimated for specific classes of sources (El-Badry et al. 2021c).

One can try to mitigate these spurious astrometric solutions with cuts on ruwe, visibility_periods_used and apparent $G$ magnitude. These cuts are known to exclude many valid sources and also bias the sky coverage. In the GCNS, the approach was to use several astrometric quality indicators to train a random forest classifier on a good and a bad training sample. Since the GCNS only contains sources with observed parallax of greater than 8 mas, it primarily covers the extremely high parallax signal-to-noise ratio (SNR) regime. For the "bad" examples, sources with measured parallax less than -8 mas were used, exploiting the fact that spurious astrometric solutions can be expected to scatter equally above and below the true parallax. Sources with extremely negative measured parallaxes can therefore be taken to be representative of sources with spurious, positive measured parallaxes. For the "good" examples, sources in low-density regions of the sky that were cross-matched to 2MASS and that showed consistent absolute magnitudes in Gaia and 2MASS bands with the locus of the main stellar populations (e.g. main-sequence or white-dwarfs) were used.

When trying to classify the spurious parallax solutions for the whole Gaia eDR3 catalog, one also needs to make informed decisions for low parallax SNR, as $85 \%$ of sources fall into this regime (for $|\mathrm{SNR}|<4.5$ ). Since low-SNR parallaxes less stringently constrain distance, it is more difficult to establish a genuine difference between valid and spurious astrometric solutions. We aim to mitigate this difficulty by training specialised models for the high- and low-SNR regimes.

In the following, we will attach a single scalar measure of "astrometric fidelity", bound between 0 and 1, to all sources in eDR3. This will prove useful when, for example, trying to clean CAMDs (see Section 5.7.2). Contamination in CAMDs can also result from spurious colors, which our astrometric fidelity measure is not intended to flag. However, we will show in Section 6 how these can be filtered out as well.

\section{OPEN SCIENCE APPROACH}

We previously submitted a preprint to arxiv. org, with a first version (v1) of our astrometric fidelities uploaded to the German Astrophysical Virtual Observatory (GAVO), in order to collect feedback from the community. Now, in the updated version (v2) of this work, we have implemented improvements that we will highlight, together with the general procedure in the following sections. We will also compare $\mathrm{v} 1$ to $\mathrm{v} 2$ and look at the improvements.

All of the work in this paper, including the training of the classifier and the various validation tests, can be replicated using a Python notebook and data that we have made available. ${ }^{1}$. This notebook should have legacy value for the community, as our analysis can be easily repeated for upcoming data releases. Additionally, others can augment our training data with extra sets of good or bad astrometric solutions, in order to improve the classifier or to produce special purpose classifiers for specific stellar populations or regions of the sky with specific properties (e.g. high density areas).

\section{TRAINING DATA}

In order to train a classifier that distinguishes between sources with reliable and unreliable astrometric solutions, we first must construct a training sample of sources that we can identify as falling into each category. We will refer to spurious (valid) astrometric solutions as "bad" ("good"). In the following, we describe our method of identifying both bad and good training sets.

In all of the following, we neglect the Gaia parallax zero-point offset (Lindegren et al. 2021b), which should be safe for most of the sources, as its magnitude is on the order of $20 \mu$ as.

\subsection{Spurious sources}

\subsubsection{Large negative parallaxes}

We obtain the bulk of our bad training sample by selecting sources with parallax_over_error $<-4.5$. We use the following query:

SELECT *

FROM gaiaedr3.gaia_source

WHERE parallax_over_error $<-4.5$

This returns 4.18 million sources. If all of the 1.47 billion sources in Gaia eDR3 with measured parallaxes had a true parallax of zero, and all of the measurement errors were Gaussian, then we would expect approximately 5000 stars - nearly three orders of magnitude fewer - to satisfy the above cut. Since in reality, sources have positive true parallaxes, the discrepancy is even larger. Thus, even with the most pessimistic assumptions, the contamination rate of our bad training sample by sources with good astrometric solutions is $\sim 0.1 \%$.

Fig. 1 shows the distribution of our bad sources over the sky. Crowded areas such as the bulge, disc and the Magellanic clouds stand out, but scanning-law patterns are also visible, with regions of the sky that are scanned most often (notably the two rings along ecliptic latitude $\approx \pm 45^{\circ}$ ) having higher densities of spurious astrometric solutions. We conjecture that this is due to the many scans

1 The notebook can be found at https://colab.research.google. com/drive/11PzhGSSIj x2nQ7XM2v8bQZtkf0Atrk0z?usp=sharing, while the necessary training and validation data is stored at https://keeper.mpdl .mpg.de/d/21d3582c0df94e19921d/ 


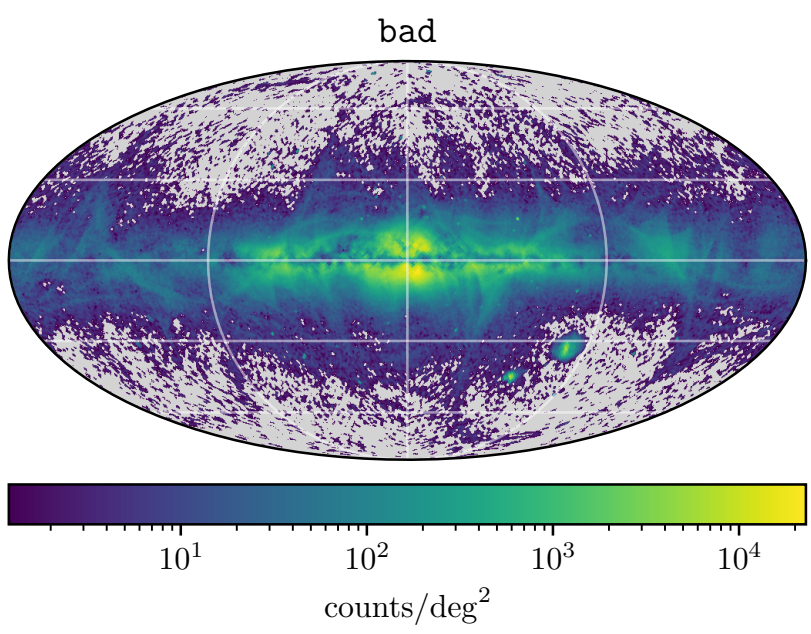

Figure 1. Density distribution of the sources identified as bad for our training sample by their highly negative $(<-4.5 \sigma)$ parallaxes, shown using a Mollweide projection in Galactic coordinates, with a logarithmic color scale.

along a similar scanning angle, which on the one hand could increase the probability of spurious detections ocurring at the same place and therefore reduce the probability of these sources being filtered out in the downstream process (Torra et al. 2021) and on the other hand could amplify the disturbance from a close neighbor.

\subsubsection{Iterative identification of spurious parallaxes}

Thus far, all of our bad training examples are in the high-SNR regime. We follow an iterative approach to obtain a sample of sources in the low-SNR regime with unreliable parallaxes. We first train our model without any low-SNR bad training examples. We then classify all GCNS sources (i.e., all source with observed parallax greater than 8 mas) and compare to the GCNS classifications. We are particularly interested in the sources that we classify as good and that the GCNS classifies as bad. In Fig. 2, we show the sky distribution and CAMD of such sources in the low-SNR regime. Only a negligible fraction of such mismatched sources are in the highSNR regime. In the low-SNR regime, the sky distribution of these sources is highly concentrated in the Galactic disk, and even contains overdensities in the directions of the Magellanic clouds. This indicates that a large fraction of these sources are likely spurious (cf. Fig. 1).

We therefore add the $\sim 23 \mathrm{k}$ low-SNR sources that the GCNS classifies as bad and our initial model classifies as good to our bad training sample, and then retrain the model. Because of the relatively small size of this sample, we assign 10 times the normal weight to these sources during training.

\subsection{Good sources}

We combine three different datasets in order to construct the good training set. The primary dataset consists of sources that lie in regions of the sky devoid of bad sources (i.e., regions that lack sources with significantly negative parallaxes). The second dataset consists of sources near the Galactic plane that lie on the main sequence in Pan-STARRS 1 (Chambers et al. 2016, "PS1") colorcolor and color-absolute-magnitude (using Gaia parallaxes) space. The third dataset consists of globular cluster members that have measured parallaxes consistent with the globular cluster parallax. By combining these different datasets, we hope to obtain a sample of good sources from a range of different regimes (e.g., source density and sky position). Additionally, in constructing the good training set, we do not cut on any astrometric flags that we use later as potential features in the classifier - doing so would render the classifier's job trivial. Similarly, as can be seen in Fig. 3, where the CMDs of the good and the bad training set are shown, both training sets have a large overlap in magnitude and color space ${ }^{2}$, with an exception for very red colors. These considerations should allow our classifier to more effectively generalize from our training dataset to the entire Gaia eDR3 catalog.

These three datasets are described in detail below.

\subsubsection{Regions without bad sources}

In order to construct our primary good training set, we select all sources with parallax_over_error $>-3$ in regions of the sky that do not contain any sources with significantly negative parallaxes. This means that our primary good training dataset and our bad training dataset come from disjoint regions of the sky. This good sample does not come from the Galactic plane (i.e., $|b|>19^{\circ}$ for all good sources). In detail, we separately query each HEALPix (Górski et al. 2005) level-6 (i.e., nside = 64) pixel that contains no sources with parallax_over_error $<-3$.6. We use a stricter cut in sparser regions of the sky, omitting HEALpix pixels with a source count below 3500 that contain any sources with parallax_over_error $<-2.7$. In all, we use 1614 out of 49152 HEALPix level-6 pixels on the sky to construct our primary good training set. Our query for a single pixel is as follows:

\section{SELECT *}

FROM (SELECT dr3.*, ps1.original_ext_source_id as obj_id, FLOOR(dr3.source_id/140737488355328) as hpx6

FROM gaiaedr3.gaia_source as dr3

JOIN gaiaedr3.panstarrs1_best_neighbour AS ps1

USING (source_id)

WHERE source_id BETWEEN O AND 562949953421311) AS subquery

-- Query only first HEALpix of level 6

JOIN gaiadr2.panstarrs1_original_valid AS ps

USING (obj_id)

-- only sources with a crossmatch to PS1 are queried

We obtain a total of 4.85 million sources. The requirement that the source also be visible in PS1 ensures that we do not include spurious Gaia sources (though the PS1 xmatch does not help in filtering spurious astrometric solutions).

We split our primary good training set into two subsets: a high-SNR subset with parallax_over_error (SNR) $>4.5$ and a low-SNR sample with $-3.0<$ SNR $<4.5$. In order to further purify our primary good training set, we require $G-R P<1.8$ mag (1.5 mag) for the high-SNR (low-SNR) subset. The cut on $G-R P$ requires $R P$ photometry and removes about $1 \%$ of unphysically red sources. This extremely red color sometimes coincides with nearby sources and/or high phot_bp_rp_excess_factor. After

2 In v1, when we crossmatched to 2MASS instead of PS1, we had a deficit of blue, faint good training sources. Explicitly below the line connecting $\left(G-R P, m_{G}\right)=(0.5,18)$ and $(1.2,20)$, we had no good training sources. The drop in density for sources brighter than $G=12 \mathrm{mag}$ is due to the saturation limit of PS1 
GCNS bad, initial model good, $\mathrm{SNR}<4.5$

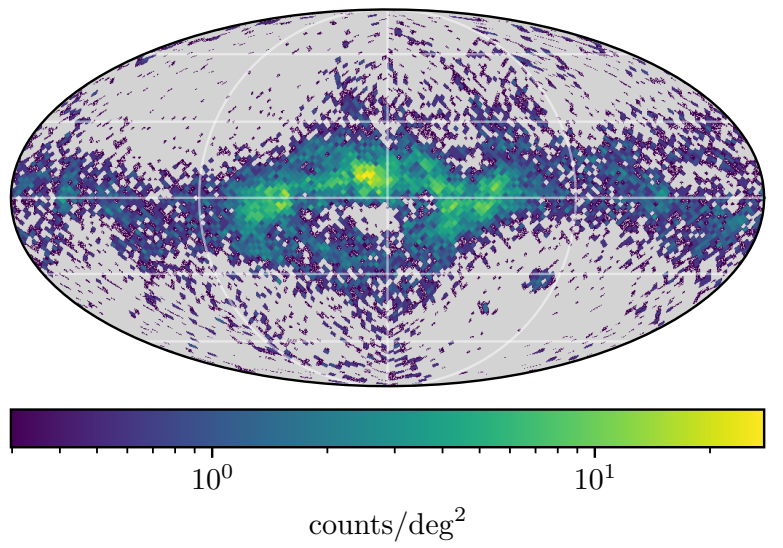

GCNS bad, initial model good, SNR $<4.5$

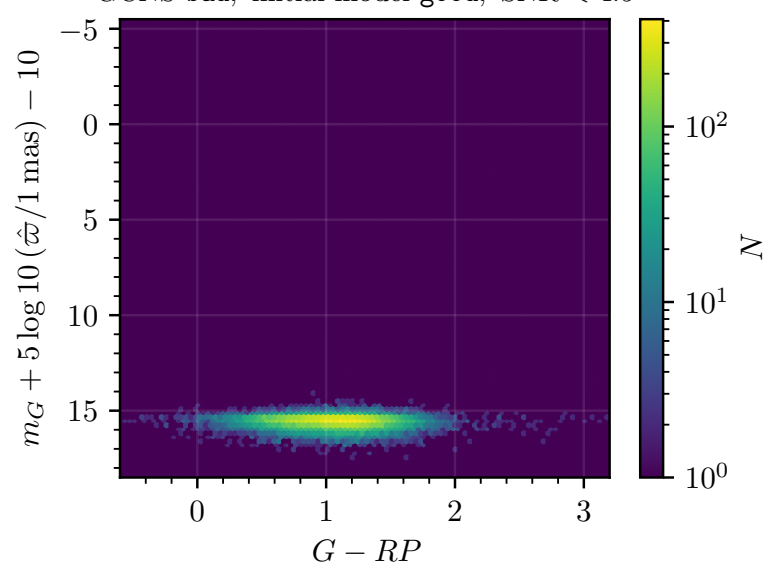

Figure 2. Sky distribution (left panel, using a Mollweide projection of Galactic coordinates) and CAMD (right panel) of the low-SNR GCNS sources ( $\hat{\varpi} \geq 8$ mas) identified as good by our initial model but as bad by the GCNS, using a logarithmic color scale. These sources are concentrated in the Galactic plane, and even show overdensities in the LMC and SMC. We use these sources to create an additional bad dataset, on which our final model is trained.

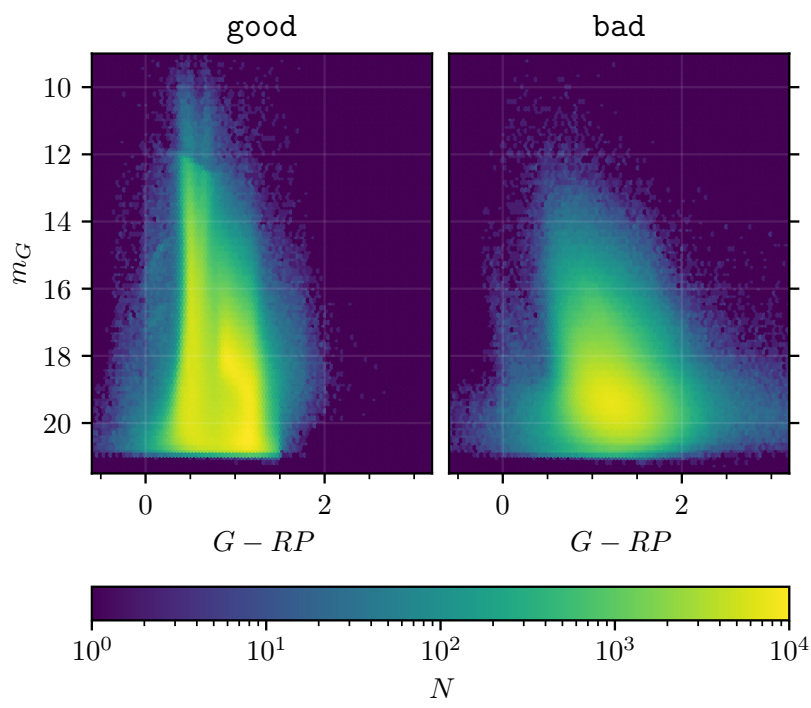

Figure 3. Color-magnitude diagram (CMD) distribution of the good (left panel) and bad (right panel) training samples, on a logarithmic color scale. The left panel shows all 6.25 million good sources (Section 3.2), while the right panel shows all 4.20 million bad sources (Section 3.1). The left panel shows the well-established and expected bimodal structure in the color distribution that arises from the main sequence turn-off and the red giant branch. The right panel shows not a hint of such structure, implying that the colors of these bad sources may commonly be spurious, too.

these photometric cuts, our primary good training set contains 4.82 million sources.

Of course, our bad and primary good training sets probe quite different regimes, with the primary good training set coming mostly from low-extinction regions and sparse fields at high Galactic latitudes. We therefore add in two additional good training sets, described in the following subsections, which are tailored to cover crowded regions of the sky. We hope (and later verify) that in the space of astrometric parameters and quality flags, our training sets cover the relevant feature space and will allow our classifier to have discriminative power over the entire sky, as was the case for the GCNS.

\subsubsection{Sources lying on the main sequence}

In order to obtain sources with well measured parallaxes nearer to the Galactic plane, we make use of a combination of PS1 photometry and Gaia parallaxes to select sources that lie on the stellar main sequence. We make use of PS1 photometry, instead of Gaia photometry, due to the increased contamination of Gaia $B P-R P$ colors in crowded regions of the sky.

On a CAMD, stars with PS1 $r-i>0.35$ that have well measured, high-SNR parallaxes should mostly lie on the main sequence, while stars with spurious parallax measurements will be scattered off of the main sequence. Using stars with high-SNR parallax measurements near the Galactic North Pole $\left(|b|>80^{\circ}\right)$, we construct a mask on the PS1 $M_{g}$ vs. $r-i$ CAMD, as well as a mask on the $g-r$ vs. $r-i$ color-color diagram, defining the main sequence, as illustrated in Fig. 4. We then select stars in the region $0^{\circ} \leq \ell \leq 80^{\circ},|b|<20^{\circ}$ that have high-SNR parallax measurements (parallax_over_error $>4.5$ ) and ruwe $<1.2$, and which lie on our CAMD and color-color main-sequence masks, based on their measured Gaia eDR3 parallaxes and extinction-corrected PS1 magnitudes. For our extinction correction, we use the mean Bayestar19 (Green et al. 2019; Green 2018) extinction estimate at the source distance (computed naively as 1 mas kpc/parallax). In order to limit the effect of the extinction correction on our selection, we exclude regions of the sky for which the Schlegel et al. (1998) reddening estimate exceeds $\mathrm{E}(B-V)=0.5 \mathrm{mag}$.

This procedure yields $980 \mathrm{k}$ sources, which we use as our second good dataset. This dataset covers crowded regions closer to the Galactic plane, helping to balance our good training set.

\subsubsection{Globular cluster sources}

Vasiliev \& Baumgardt (2021) ("VB21") found that the initial version (v1) of our classifier underperformed in globular clusters. This is most likely due to the fact that our primary good training examples come from sparse regions of the sky, while our bad training examples are drawn primarily from dense regions of the sky. 

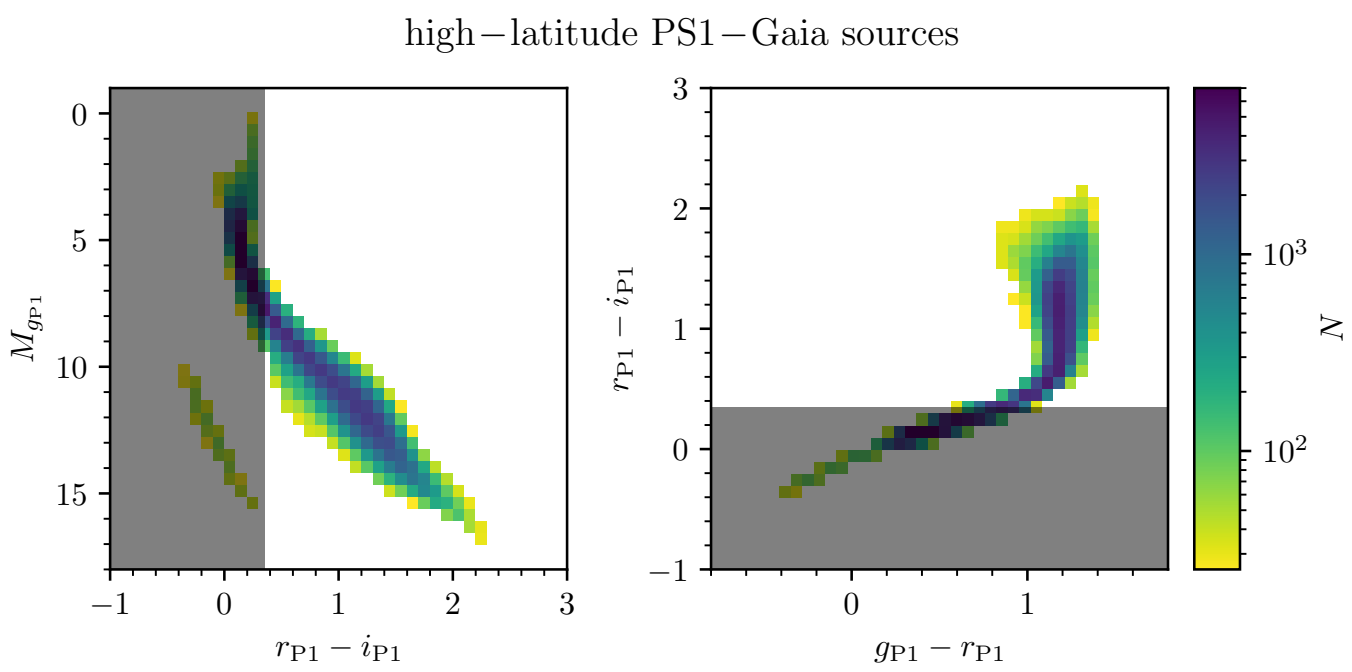

Figure 4. The left panel shows a PS1-Gaia CAMD (at high Galactic latitudes), using PS1 photometry (de-reddened using Bayestar19) and high-SNR Gaia parallaxes. We use stars with $r_{\mathrm{P} 1}-i_{\mathrm{P} 1}>0.35$ (the unshaded region) to define a main sequence mask on the CAMD. We additionally construct a PS1 color-color mask (right panel). We then use these CAMD and color-color templates to select stars in the Galactic plane with good Gaia astrometry.

In order to obtain good training data in the most crowded regime, we make use of globular cluster members identified by VB21. In detail, we select sources identified by VB21 as having high cluster membership probability $(>90 \%)$, which additionally have measured parallaxes consistent with their respective host-cluster parallax (as determined by VB21, i.e., their qflag $\geq 2$ ). This procedure results in $450 \mathrm{k}$ good sources.

\section{TRAINING THE CLASSIFIER}

We train a neural network to distinguish between our good and bad training sets, using a set of features that come from the Gaia Archive, as well as information about the neighborhood of each source. In the following, we describe our training features, the architecture and training of the neural network, and finally compare our neural network to simpler classifiers.

\subsection{Training features}

Generally, we want a set of features that enable our classifier to distinguish between good and bad sources, while avoiding features that will allow our classifier to trivially distinguish between the training sets, but which would not generalize to the entire Gaia eDR3 catalog. As the GCNS obtained good classification results for nearby stars, we use a similar set of features as that work (marked in gray in Table A.1 of Gaia Collaboration et al. 2021b). In detail, we use the following features from the Gaia EDR3 gaia_source table: ${ }^{3}$

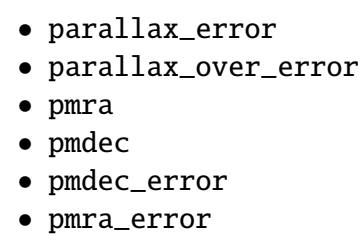

${ }^{3}$ See the Gaia eDR3 data model for details on what each feature represents.
- astrometric_sigma5d_max

- astrometric_excess_noise

- visibility_periods_used

- ruwe

- astrometric_gof_al

- ipd_gof_harmonic_amplitude

- ipd_frac_odd_win

- ipd_frac_multi_peak

- matched_transits_removed

- astrometric_params_solved

- astrometric_excess_noise_sig

Critically, we take the absolute value of parallax_over_error before passing it to the classifier, as our bad training contains only strongly negative parallax_over_error values. Giving the classifier information about the sign of the parallax measurements would render classification of the training set trivial, but would not generalize to the entire Gaia survey, in which spurious parallaxes can be both positive or negative. We additionally take the absolute value of pmra and pmdec, as the direction of the proper motions might carry information about the location of a star on the sky - information we do not want to give the classifier, given the spatially disjoint nature of our bad and primary good training sets ${ }^{4}$. Finally, and perhaps trivially, the actual source sky position is of course not used in the classification.

From Lindegren et al. (2021b), we know that astrometric solutions with different numbers of parameters have systematic differences. We therefore add astrometric_params_solved to the feature list. This feature was neither used in v1 nor in the GCNS paper. Similarly, matched_transits_removed is also new. Both parameters depend on information from Gaia DR2 that might not be available due to a new or changed source_id (which can happen without any relation to the astrometric solution). Tests showed that this does not reduce the performance of our classification, probably because other features still indicate the quality of the astrometric

4 The total proper motion would be a possible replacement for pmra and pmdec. However, following GCNS, we use the individual vector components. 
solution and the training sets will contain enough examples such that the classifier can still be safely generalized.

In addition to the gaia_source table columns, for each source, we calculate the distance to the nearest source that is at least $X$ mag brighter, for various values of $X$. We call these features dist_nearest_neighbor_at_least_X_brighter, where $\mathrm{X} \in\{0,2,4,6,10\}$. The presence of nearby, bright neighbours is plausibly one of the largest causes of bad astrometry or colors (see Section 6), ${ }^{5}$ and is therefore potentially a powerful feature for identifying spurious astrometric solutions. These features were not used by the GCNS. In the development of our classifier, we additionally explored the use of fainter nearby neighbors (i.e., neighbors with $X<0$ ), but found that they do not significantly improve the overall performance of the classifier, beyond what we achieve with the above features. As information about faint neighbors might simply be a proxy for overall density of sources on the sky, we decided against providing it to the classifier. We truncate dist_nearest_neighbor_at_least_X_brighter at 5 arcsec, as distant neighbors should have no appreciable effect on parallax measurements, and any inferences drawn from the presence of more distant neighbors would likely reflect idiosyncrasies of our training data (e.g., most of our good samples come from low-density regions of the sky) and generalize poorly to the entire eDR3 catalog. Our table of neighbor distances is available together with our v1 and v2 fidelities from GAVO. We also calculate and report a metric of expected color contamination that accounts for both magnitude difference and angular separation, as described in Section 6.

\subsection{Training for two different $|\mathrm{SNR}|$ regimes}

We train two different classifiers, intended for use in the regimes $|\mathrm{SNR}|<4.5$ and $|\mathrm{SNR}|>4.5$ (where we use parallax_over_error as a proxy for SNR). We will refer to these classifiers as the "low-SNR" and "high-SNR" classifiers, respectively. The most important difference between these two classifiers is that the high-SNR classifier uses $|\mathrm{SNR}|$ as a feature, while the low-SNR classifier does not. Recall that our bad training set includes very few sources with $|S N R|<4.5$ (i.e., those from our iterative retraining). If we were to allow the low-SNR classifier to take $|\mathrm{SNR}|$ into account, it would learn that there are very few bad sources with $|S N R|<4.5$, which is simply an artifact of our method of identifying bad training data.

\subsection{Neural network training}

In contrast to the work on GCNS, where spurious sources were identified using a random forest, we employ a feed-forward neural network (NN) here. Our NN model consists of 4 hidden layers, each with 64 neurons and a Rectified Linear Unit (ReLU) activation. The final layer has a single neuron with a sigmoid activation, and represents the probability that a source belongs to the good class. We use the binary cross-entropy loss function (e.g. Goodfellow et al. 2016), which is closely related to the Kullback-Leibler divergence and which measures how much additional information would be

\footnotetext{
5 One could add phot_bp_rp_excess_factor as a training feature, as it also correlates with nearby sources, but this would restrict the applicability of our model to eDR3 sources which have astrometric solutions and both $B P$ and $R P$ photometry. We therefore use dist_nearest_neighbor_at_least_X_brighter as a proxy that does not need $B P$ and $R P$ photometry.
}

needed, on average, to correct the classifier's prediction. Given input features $\vec{x}$, the classifier outputs a probability $P(\vec{x})$ that the source belongs to the good class. Denote true class (the label) by $y \in\{0,1\}$ (where $y=1$ signifies "good"). The binary cross-entropy is then given by

$\mathcal{H}=-(1-y) \ln [1-P(\vec{x})]-y \ln P(\vec{x})$.

As the binary cross-entropy is a measure of information, it can be expressed in units of bits or nats (by using natural logarithms above, we have chosen to use nats).

We implement our model in Tensorflow 2 (Abadi et al. 2016) and Keras (Chollet et al. 2015). We train for 150 epochs with an Adam optimizer (Kingma \& Ba 2014), using a learning rate of $10^{-3}$ in the first 50 epochs, a learning rate of $10^{-4}$ in the subsequent 50 epochs, and a learning rate of $10^{-5}$ in the final 50 epochs. During training, we apply a dropout rate of 0.1 after each hidden layer in order to avoid over-fitting. The features are normalised (to zero mean and unit variance) prior to the training.

We set aside $10 \%$ of the data as a "test" dataset, which we use to assess the performance of our final, trained model. Of the remaining training data, we set $20 \%$ aside as a "validation" dataset, which we use to assess progress during the training procedure. Thus, in total, $72 \%$ of our data is used for training, $18 \%$ is used for validation during training, and $10 \%$ is used to test the performance of the final model.

We train our high- and low-SNR classifiers separately. For both classifiers, our validation loss is lower than our training loss (due to the use of dropout during training), indicating that we are not over-fitting. For both classifiers we do not restrict the good training sample to the respective SNR range. This reduces slightly the performance measured with the test set (that was drawn from the training sample), but improves performance when applying the classifier to independent validation sets, i.e. generalizes better to the whole eDR3.

As explained in Section 3.1.2, after training our model, we find that low-SNR sources that GCNS classifies as bad but ours as good have an implausible on-sky distribution. We incorporate these sources into our bad training sample and re-train the model. In the remainder of the paper, we report the results with this retrained model.

We assess our final performance by applying the low-SNR classifier to our low-SNR test dataset, and our high-SNR classifier to our high-SNR test dataset. On this combined test dataset, we achieve a loss of 0.0571 nats of cross-entropy, with a purity of $99.3 \%$ and a completeness of $97.3 \%$. On the high-SNR dataset, we achieve a binary cross-entropy of 0.0457 nats, with a purity of $98.9 \%$ and a completeness of $97.2 \%{ }^{6}$. This performance is an upper limit for real world applications, as our training samples are fairly well separated in feature space. Particularly in the low-SNR regime, it is difficult to discern good from bad astrometric solutions.

In Fig. 5, we show histograms of the predicted astrometric fidelity for good and bad sources in the test dataset. In the rest of this work, we classify objects with fidelity $>0.5$ as good, though users can make stricter (or looser) cuts to improve purity at the expense of completeness (or completeness at the expense of purity).

\footnotetext{
${ }^{6}$ For comparison, when using an ExtraTree (Geurts et al. 2006) classifier (which is closer in performance to the GCNS paper's random forest) in the high-SNR regime, we obtain a purity of $93.7 \%$ and a completeness of $95.7 \%$, showing the superior performance of the neural net classifier.
} 


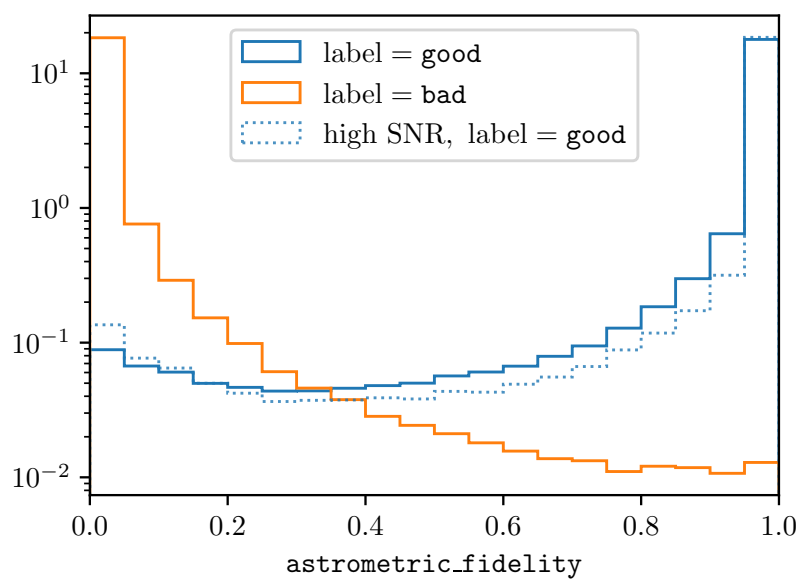

Figure 5. Histogram of the predicted classifier probabilities (of belonging to the good class) for sources in the test dataset, split by training label. As the good class contains both low- and high-SNR training data, we additionally show the classifier probabilities for the high-SNR good sources. The $x$-axis represents the probability output by the classifier that a given source is good, which we term the "astrometric fidelity."

\subsection{Comparison with simpler classifiers}

Here, we compare the astrometric fidelity predicted by our neural network to analogous quantities obtained using simpler classifiers. First, we evaluate how cleanly simple cuts on ruwe and astrometric_excess_noise separate good and bad sources in the high-SNR test dataset. Fig. 6 shows the binary crossentropy, purity and completeness of the cut as a function of the threshold value for each feature. For ruwe, we achieve a minimum binary cross-entropy of 1.51 nats using a cut of ruwe $<1.11$, corresponding to a purity of $87.0 \%$ and a completeness of $88.0 \%$. For astrometric_excess_noise, we achieve a minimum binary cross-entropy of 1.14 nats for a cut of astrometric_excess_noise $<0.68$, corresponding to a purity of $90.7 \%$ and a completeness of $90.3 \%$.

Next, we train a logistic model, which takes into account a linear combination of features. This model assigns a probability

$P(\operatorname{good} \mid \vec{x})=\left[1+e^{-(\vec{w} \cdot \vec{x}+b)}\right]^{-1}$

of belonging to the good class to each source, where $\vec{x}$ is a vector containing the features, $\vec{w}$ is a vector containing a weight for each feature, and $b$, the bias, is a scalar. We use the Adam optimizer to find the weights and bias that minimize the binary cross-entropy of the predictions. On the high-SNR test dataset, we obtain a binary cross-entropy of 0.100 nats, a purity of $95.7 \%$ and a completeness of $95.9 \%$. This is better than what we achieve with simple cuts, but still represents nearly twice the binary cross-entropy we obtain with the full neural network model.

The full neural network is not significantly more difficult to implement than these simpler classifiers, and it achieves a far more complete and pure separation of the test dataset. For these reasons, we strongly favor use of the full neural network classification over simpler alternatives.
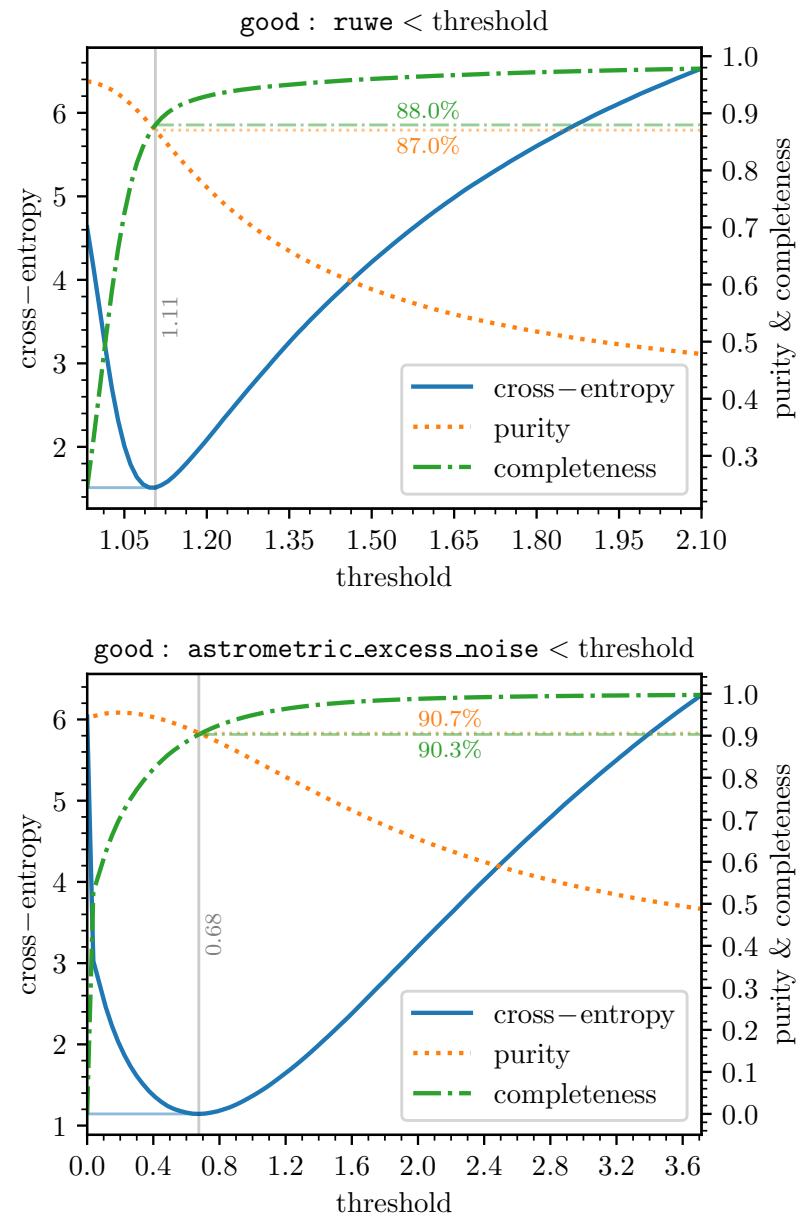

Figure 6. Performance of simple cuts on ruwe (top panel) and astrometric_excess_noise (bottom panel) in differentiating good and bad astrometric solutions. In each panel, we show how binary cross-entropy (Eq. 1), purity and completeness depend on the threshold chosen for the cut. Our neural network predicting astrometric fidelity achieves an order of magnitude less contamination $(99.3 \%$ purity and $97.3 \%$ completeness see Section 4.2) than the optimal choices for these cuts (at minimal crossentropy).

\section{OUTSIDE VALIDATION}

Our classifier performs very strongly on the test dataset, which is statistically identical to the training data. In this section, we perform several tests using a variety of outside datasets, in order to determine whether our classifier generalizes beyond our training data. Here, the validation revolves around external information on the distance (e.g. membership in a cluster or the Large Magellanic Cloud), the plausibility of the distribution of sources on the sky or in the disk of the Milky Way, or simply external measurements (e.g., comparison of Gaia proper motions with those measured by other surveys).

\subsection{Gaia Catalogue of Nearby Stars}

All 1.2 million sources with parallax $>8$ mas from eDR3 have classifications from the GCNS (Gaia Collaboration et al. 2021b). Here, we measure our agreement with these classifications. As described in Section 3.1.2, we train two iterations of our model, using a sample of low-SNR sources from the GCNS in our bad training sample in the second iteration. Thus, our final model is influenced 


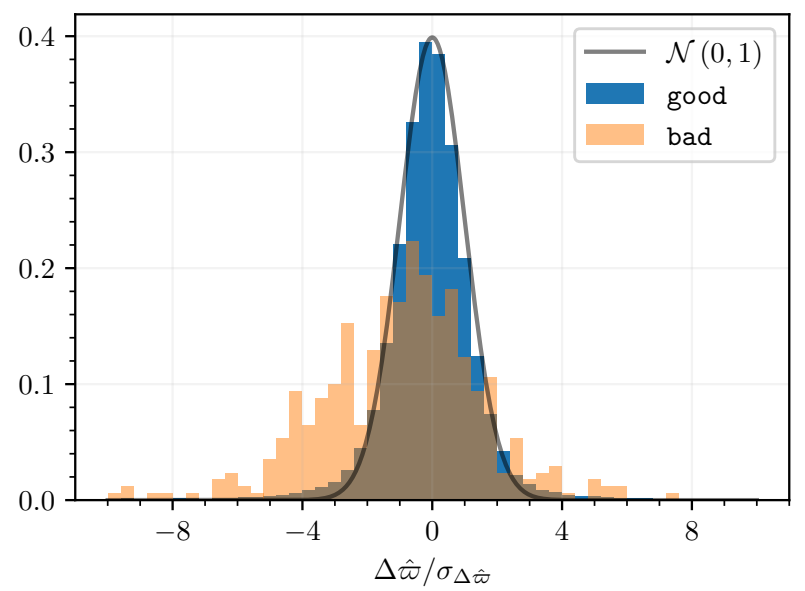

Figure 7. Validation of our astrometric fidelity prediction using open and globular clusters. The figure shows histograms of the standardized parallax residuals for good and bad sources. The true parallaxes are estimated using the variance-weighted mean of the parallaxes in each cluster. We restrict this comparison to clusters with distances determined to $20 \%$ or better. The good sources closely follow the expected unit normal distribution, in marked contrast to the standardized residuals of the bad sources.

by the GCNS classifications, and will - by construction - have good agreement.

We therefore report our agreement with GCNS both for our initial and for our final model. Taking the GCNS classifications as ground truth, our initial model achieves a purity of $96.0 \%$ and a completeness of $99.4 \%$. Our final model achieves a purity of $99.7 \%$ and a completeness of $99.3 \%$.

\subsection{Clusters}

Open and globular clusters, and the "prior" information on the distance of their likely member stars, offer a great opportunity to validate our parallax classifier. We begin with a catalog of 162,484 sources assigned to 121 clusters, coming from Bailer-Jones et al. (2018). This catalog was compiled using a method similar to that used in Gaia Collaboration et al. (2018). For each individual cluster, we calculate the variance-weighted mean parallax of its member sources, as well as the corresponding uncertainty in the mean parallax:

$\langle\hat{\varpi}\rangle=\sum_{i} \frac{\hat{\varpi}_{i}}{\sigma_{i}^{2}}, \quad \sigma_{\langle\hat{\varpi}\rangle}=\left(\sum_{i} \frac{1}{\sigma_{i}^{2}}\right)^{-1 / 2}$.

We then select the 41 clusters for which $\sigma_{\langle\hat{\omega}\rangle} /\langle\hat{\varpi}\rangle<0.2$ (i.e., for which parallax is determined to better than $20 \%$ ). For each source in each of these clusters, we then calculate a parallax residual, using the estimated cluster parallax as a reference:

$\Delta \hat{\varpi} \equiv \hat{\varpi}-\langle\hat{\varpi}\rangle, \quad \sigma_{\Delta \hat{\varpi}}=\left(\sigma_{\hat{\varpi}}^{2}+\sigma_{\langle\hat{\varpi}\rangle}^{2}\right)^{1 / 2}$.

The distribution of these parallax residuals (divided by the corresponding uncertainties) is shown in Fig. 7. Our classifier labels approximately $1 \%$ of sources in these clusters as bad. The standardized residuals of sources classified as good roughly follow the expected unit normal distribution, while the distribution of standardized residuals of the sources classified as bad is shifted negative and has much longer tails.

\subsection{OGLE proper motions}

The Fourth Phase of the Optical Gravitational Lensing Experiment (OGLE-IV, Udalski et al. 2015) began observing the bulge of the Milky Way in 2010. Here, we validate our classifier using sources with proper-motion measurements from both OGLE-IV (OGLE Uranus astrometry project, Udalski et al. 2021, in preparation) and Gaia eDR3. Our assumption is that objects with spurious parallax determinations in Gaia eDR3 are more likely to have spurious proper-motion determinations. This should be reflected in the proper-motion residuals between Gaia eDR3 and OGLE-IV, with sources classified as bad in Gaia eDR3 having systematically higher $\chi^{2}$ values in this comparison. We begin with a cata$\log$ of OGLE-IV sources with proper-motion measuresments, lying in a $0.15 \mathrm{deg} \times 0.15 \mathrm{deg}$ box centered on $\left(\alpha_{\mathrm{J} 2000}, \delta_{\mathrm{J} 2000}\right)=$ (271.761 deg, $-26.698 \mathrm{deg}$ ). Using a matching radius of $0.2^{\prime \prime}$, we obtain 14125 matching Gaia eDR3 sources with measured proper motions. Our classifier labels 2870 of these sources good.

We calculate the proper-motion residuals, $\Delta \vec{\mu} \equiv \vec{\mu}_{\mathrm{Gaia}}-$ $\vec{\mu}_{\text {OGLE}}$, as well as the covariance matrix of the residuals, $C_{\Delta \vec{\mu}}=$ $C_{\mu \text {, Gaia }}+C_{\mu \text {, OGLE }}$. We then calculate $\chi^{2}=\Delta \vec{\mu}^{T} C_{\Delta \vec{\mu}}^{-1} \Delta \vec{\mu}$ for each source. If the uncertainties are well estimated and the residuals follow a Gaussian distribution, then the $\chi^{2}$ values that we obtain should follow a $\chi^{2}$ distribution with two degrees of freedom. However, we find that the resulting $\chi^{2}$ values are significantly larger, on average, than expected, both for sources labeled good and bad, indicating that Gaia eDR3 and/or OGLE-IV proper-motion uncertainties are underestimated in the Galactic Bulge. One could attempt to address this problem by inflating the uncertainties by a constant factor or by introducing a systematic error floor. However, these different methods of "correcting" the proper-motion uncertainties impact the distributions of $\chi^{2}$ values obtained for the good and bad sources differently, as the good sources tend to have smaller estimated proper-motion uncertainties than the bad sources. In order to avoid these difficulties, we restrict our comparison to sources in a relatively small range of estimated proper-motion uncertainties, for which we assume the true proper-motion uncertainties to be similar. In particular, we select sources with $0.1 \mathrm{mas} \mathrm{yr}^{-1}<\left|C_{\Delta \vec{\mu}}\right|^{1 / 4}<0.2 \mathrm{mas} \mathrm{yr}^{-1}$, obtaining 1503 sources labeled good and 1657 sources labeled bad. The resulting distributions of $\chi^{2}$ values are displayed in Fig. 8. We find that sources labeled good by our classifier tend to have significantly lower $\chi^{2}$ values than those labeled bad, with the median $\chi^{2}$ per degree of freedom (dof) for the good subsample being 4.4, and the median $\chi^{2} /$ dof of the bad subsample being 7.9.

\subsection{Large Magellanic Cloud}

In the direction of the Large Magellanic Cloud (LMC), the vast majority of sources should be at a distance of $\sim 50 \mathrm{kpc}$, corresponding to a parallax of 0.02 mas. This affords us another opportunity to validate our classifications, as almost all stars labeled good in this region of the sky should have reported parallaxes consistent with 0.02 mas. We expect the bad sources to have larger than reported residuals, and to scatter equally to positive and negative parallaxes, leading to a widened distribution of reported parallaxes centered on 0.02 mas.

We query a 0.25 deg cone in Gaia eDR3, centered on Galactic coordinates $(\ell, b)=(280.47 \mathrm{deg},-32.88 \mathrm{deg})$, obtaining 252,115 sources, which we then run through our classifier. In this densely crowded region of the sky, only $16.05 \%$ of all sources are classified as good, while only $0.7 \%$ of high-SNR sources are classified as 


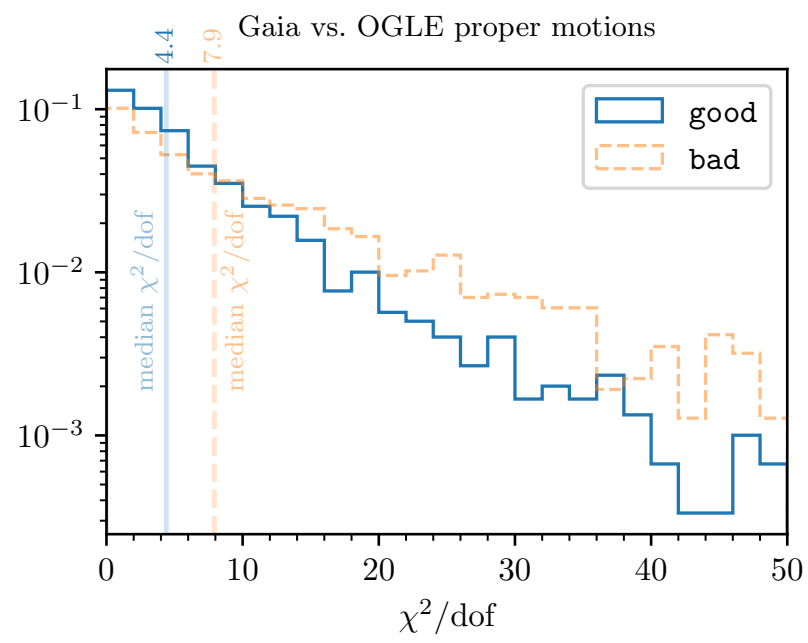

Figure 8. Validation of our astrometric fidelity classification through proper motion comparison between OGLE and Gaia eDR3. Shown is the distribution of $\chi^{2} /$ dof, based on a comparison of Gaia eDR3 and OGLE-IV proper motions in the Galactic Bulge, for sources labeled good and bad by our classifier. For ideal data, the median $\chi^{2} /$ dof would be $\sim 0.69$. We find that that proper-motion uncertainties are underestimated for Gaia eDR3 and/or OGLE-IV, leading to larger $\chi^{2} /$ dof values. However, for sources labeled good by our classifier, Gaia eDR3 and OGLE-IV proper motions match significantly better, as indicated by the lower median $\chi^{2}$ /dof values ( 4.4 for the good subsample, vs. 7.9 for the bad subsample).

good. In order to model the small number of Milky Way foreground stars in this field, we compare to a control field of the same apparent size with the same Galactic latitude, and longitude reflected around $\ell=0 \mathrm{deg}$. This control field has 1589 sources. In the control field, $85.3 \%$ of the sources are classified as good.

Fig. 9 shows the parallax distribution of good and bad sources with small reported errors (parallax_error $<0.2$ ) in our LMC field. The parallax distribution of good sources is consistent with a distant population of stars with well-measured errors, plus a small foreground population of Milky Way stars at larger parallax (matching the control field). The bad sources are consistent with a distant population of stars with significantly underestimated parallax errors. Our classifier is thus clearly identifying sources with excess parallax residuals, and even in this dense field, is still cleanly identifying foreground stars.

\subsection{The Structure of the Galactic Disk in OBA stars}

The catalog of O-, B-, and A-type (OBA) stars devised by Zari et al. (2021) ("Z21") offers another opportunity to test our classifier with an ensemble of sources at low Galactic latitudes. Z21 selects stars brighter than $G=16 \mathrm{mag}$, with Gaia eDR3 and 2MASS colors consistent with (reddened) OBA-type stars. Z21 does not apply any condition on the parallax error, as the sample was designed to be inclusive for spectroscopic follow-up.

We run our classifier on the resulting catalog, which contains $\sim 1$ million stars. Fig. 10 shows the distribution of Z21 sources classified as good (left, $\sim 80 \%$ of the initial sample) and bad (right) in the Galactic plane $\left(|b|<25^{\circ}\right)$. The distribution of sources with good astrometric solutions shows known regions of young stars and traces the spiral arm structure of the Milky Way disk, as discussed in Z21 (cf. their Fig. 11). The distribution of sources with bad astrometric solutions shows a ring-like feature centered on the Sun,

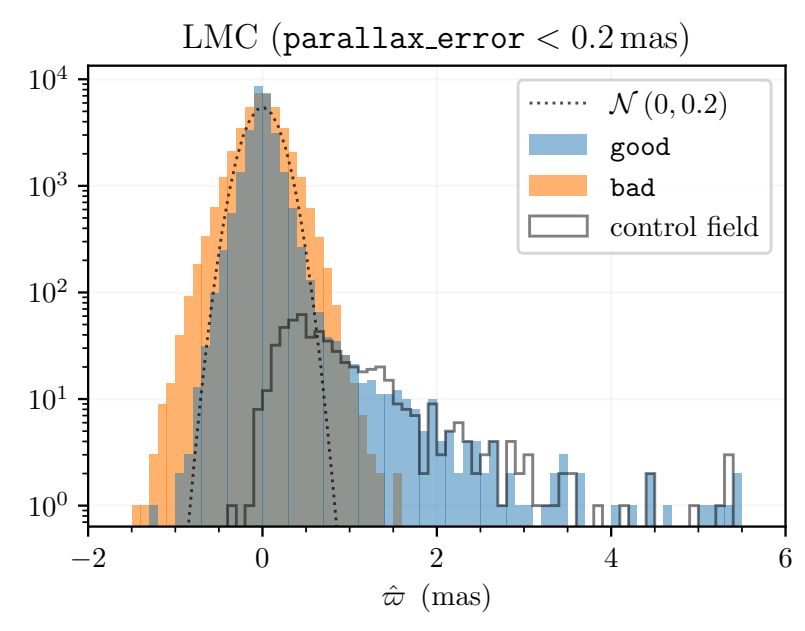

Figure 9. Validation of our astrometric fidelity classification through the parallax distribution of good and bad sources with small parallax uncertainties $\left(\sigma_{\hat{\imath}}<0.2\right.$ mas $)$ towards the LMC. The parallax distribution of good sources is consistent with a large population of distant $(\hat{\varpi} \approx 0)$ sources (approximated by a normal distribution with zero mean and a standard deviation of 0.2 mas), along with an expected population of foreground stars (matching the distribution of parallaxes in a control field). In contrast, the bad parallaxes are consistent with a distant population of stars with parallax uncertainties that are underestimated by $\sim 50 \%$. This indicates that in dense regions of the sky, our astrometric fidelities distinguish between sources with well-measured parallaxes, and sources with parallax residuals that are substantially larger than the reported uncertainties.

at a distance of 2 to $3 \mathrm{kpc}$, which is physically implausible and hence presumably spurious. This is expected, as the parallax distribution of all sources in the OBA catalog peaks at around 0.3 mas $(\sim 3 \mathrm{kpc})$.

A similar structure (called the "bloody-eye effect") was observed in Gaia DR2 by Anders et al. (2019, e.g. their Fig. A2), in which the cleaning of spurious results was achieved by a quality flag definition (STARHORSE_OUTFLAG[Q]) based on a cut in the space of relative distance uncertainty vs. $\log g$. Our astrometric fidelity classifier confirms the presence of the effect in Gaia eDR3, and resolves the issue using Gaia astrometric data alone.

\subsection{White dwarf test}

We use a spectroscopically confirmed sample of white dwarfs (WDs) from Gentile Fusillo et al. (2021) to determine how many presumably good WDs are excluded by our classifier. We only use WDs with sdss_clean $1==1$, together with type belonging to the set $\{\mathrm{WD}|\mathrm{DZ}| \mathrm{DQ}|\mathrm{DC}| \mathrm{DB} \mid \mathrm{DA}\}$. All 23k WDs should have well measured parallaxes, as their positions on the CAMD lie in the WD area. Whereas v1 of our classifier marked $7.7 \%$ of these WDs as bad, our updated v2 classifier only classifies $2.6 \%$ of the WDs as bad. This improvement is likely due to the fact that our good training data is cross-matched with PS1 (rather than with 2MASS, as in v1), which allows for fainter blue objects to enter the training sample. This test can serve as a simple estimate (upper limit) for the incompleteness that our classification may induce for high-latitude samples of sources across a wide range of apparent magnitudes.

\subsection{Source distribution in parallax bins}

As a final approach to validation, we visually inspect the projected sky distribution and CAMD of Gaia eDR3 sources classified as 

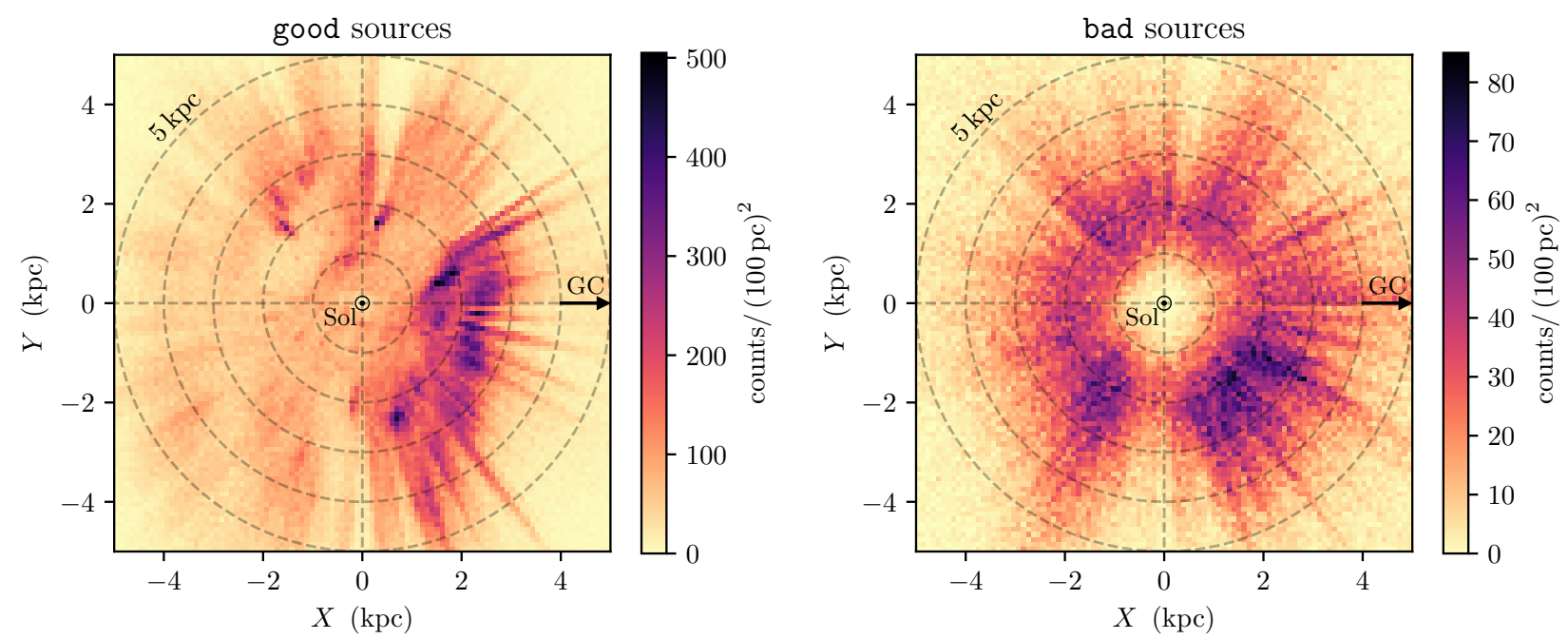

Figure 10. Validation of our astrometric fidelity classification through the astrophysical plausibility of the distribution of young stars in the Galactic plane, as identified by Zari et al. (2021). The left (right) panel shows the spatial distribution of good (bad) OBA-star sources in the Galactic plane, with the Sun located at $(X, Y)=(0 \mathrm{kpc}, 0 \mathrm{kpc})$, and the Galactic center at $(8.2 \mathrm{kpc}, 0 \mathrm{kpc})$. We have divided the plane into pixels $100 \mathrm{pc}$ on a side. The good sources show concentrations at many known locations of young stars, and show a spiral-arm-like morphology. The bad sources show a ring-like structure, exactly centered on the Sun and at the (seeming) distance of the most common parallax - clearly, far too Ptolemaic a distribution to be real.

good and bad in narrow bins of measured parallax. We additionally compare with mock observations generated using GeDR3mock (see in Appendix B for the query, where we sample the uncertainties and also use HEALPix-specific $G$-band magnitude limits).

We refer to the parallax bins by their corresponding nominal distances. The $100 \mathrm{pc}$ sample consists of the 1.2 million sources with $\hat{\varpi}>8$ mas (the GCNS sample). The 300 pc sample consists of the 1.3 million sources with 3.3 mas $<\hat{\varpi}<3.4$ mas, the $1 \mathrm{kpc}$ sample $(1$ mas $<\hat{\varpi}<1.01$ mas) contains 3.2 million sources, the $3 \mathrm{kpc}$ sample $(0.333$ mas $<\hat{\varpi}<0.334$ mas $)$ contains 1.3 million sources, the $10 \mathrm{kpc}$ sample $(0.1$ mas $<\hat{\varpi}<0.101$ mas $)$ contains 1.2 million sources, and the $30 \mathrm{kpc}$ sample $(0.0325$ mas $<\hat{\varpi}<0.034$ mas $)$ contains 1.4 million sources.

The figures we will show in the following subsections will all follow a similar pattern: The distance slices will increase from top to bottom and on the left side will be the GeDR3mock sources (our expectation for good sources), in the middle the eDR3 sources classified as good and in the right panel the ones classified as bad.

\subsubsection{Sky distribution}

We begin by looking at the sky distributions of the high-SNR sources in Fig. 11. The density is shown on a logarithmic color scale and the range of the left (GeDR3mock) and middle (eDR3 good) panel are set by the total range of the middle panel. In the middle panel we see that with increasing distance the structure of the Galactic disc is becoming more and more visible. At 10kpc the high-SNR sources peter out. Except for a higher normalization in the $100 \mathrm{pc}$ sample the GeDR3mock distribution on the left looks very similar to the eDR3 data $^{7}$. The fraction of bad sources (right panel) decreases with

\footnotetext{
7 In the 100 and $300 \mathrm{pc}$ bin the open clusters are more pronounced in GeDR3mock than in the good sources. The reason is that known cluster's true sky positions were used while masses were assigned randomly when producing GeDR3mock clusters (Rybizki et al. 2020).
}

increasing distance from $48 \%$ at $100 \mathrm{pc}$ to $3 \%$ at 3 and $10 \mathrm{kpc}$. With increasing distance it also resembles less and less the distribution of bad training sources as shown in Fig. 1 and concentrates more towards the Galactic plane.

For the low-SNR sources in Fig. 12, we expect (based on GeDR3mock) very few good sources in the $100 \mathrm{pc}$ sample ${ }^{8}$. Interestingly, we already see the imprint of the central parts of the Galaxy and of the Magellanic clouds in the good sources in the $300 \mathrm{pc}$ slice. This is expected: GeDR3mock also shows this imprint at $300 \mathrm{pc}$, due to poorly constrained (but valid) parallax measurements of sources that are, in reality, much farther away. In GeDR3mock, the visible scanning pattern is not well matched to that of eDR3. This is due to GeDR3mock uncertainties being trained on GDR2, and only roughly scaled to match eDR3 uncertainties. At $300 \mathrm{pc}$, the number of good sources we recover in eDR3 sources is almost twice as large as the number of GeDR3mock sources. This may be due to the true eDR3 uncertainties being larger than those modeled in GeDR3mock, scattering a greater number of distant sources into the $300 \mathrm{pc}$ slice. The subsequent distance bins are then relatively similar in number and actual distribution. One prominent exception is the hole in the Galactic Center that is apparent to larger distances in the eDR3 data, and which results from the lower $G$-magnitude limit of the real survey, which is not fully reflected in the GeDR3mock $G$-magnitude limit approximation (90th percentile of the eDR3 $G$ magnitude distribution, see Appendix B). The bad sources trace Galactic overdensities well, again with decreasing fractions for increasing distance bins. At $100 \mathrm{pc}$, the scanning law is still visible, but the sparsity is mainly due to most sources having a high SNR in this distance bin.

\footnotetext{
${ }^{8}$ Before adding the bad training sample from the GCNS comparison, as described in Section 3.1.2, we would have more sources classified as good in the $100 \mathrm{pc}$ sample.
} 


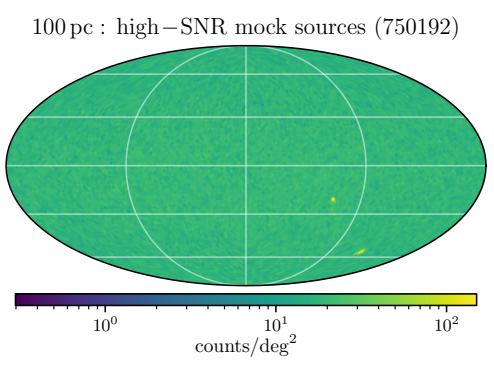

300 pc : high-SNR mock sources (491246)

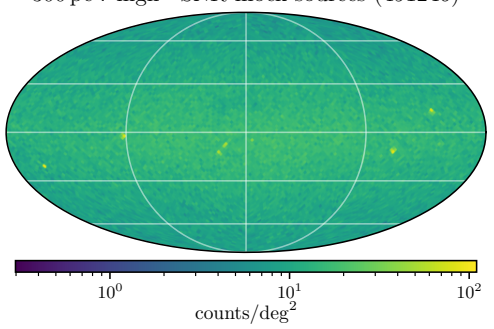

$1 \mathrm{kpc}$ : high-SNR mock sources (838010)

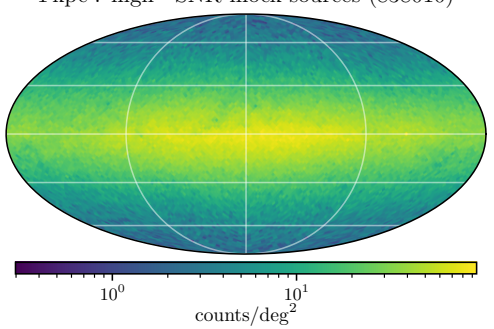

$3 \mathrm{kpc}$ : high-SNR mock sources (289482)

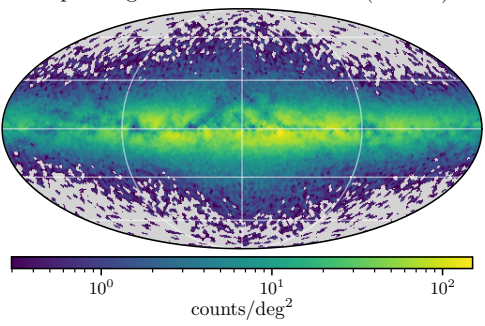

$10 \mathrm{kpc}$ : high-SNR mock sources (12512)

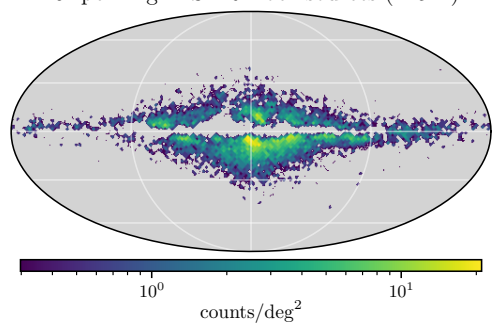

$100 \mathrm{pc}$ : high-SNR good sources (571734)

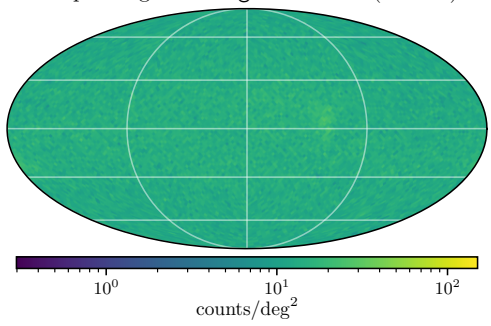

$300 \mathrm{pc}$ : high-SNR good sources (443389)

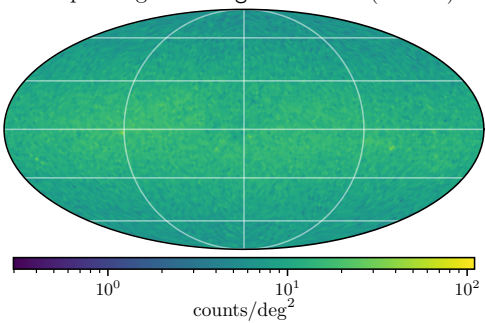

$1 \mathrm{kpc}$ : high-SNR good sources (801971)

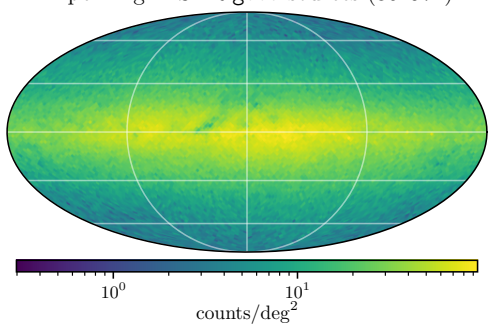

$3 \mathrm{kpc}$ : high-SNR good sources (211525)

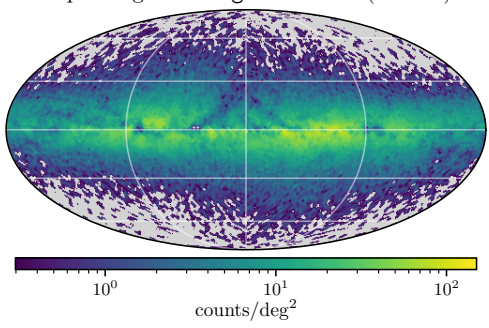

$10 \mathrm{kpc}$ : high-SNR good sources (11031)

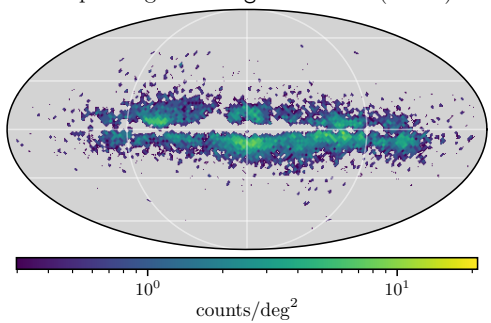

100 pc : high-SNR bad sources (535952)

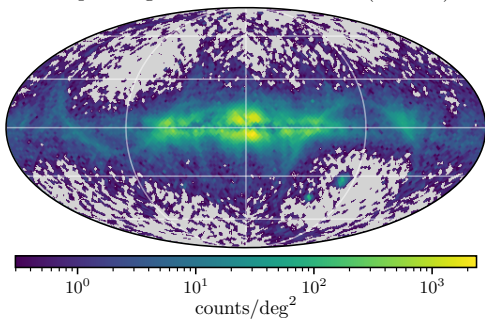

300 pc : high-SNR bad sources (144293)

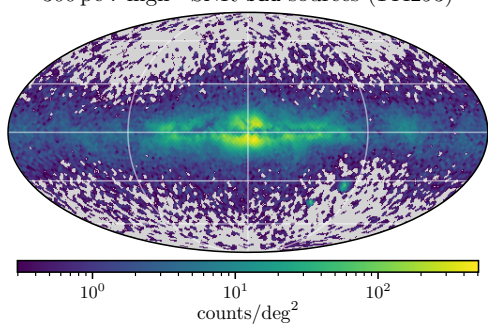

$1 \mathrm{kpc}$ : high-SNR bad sources (52149)

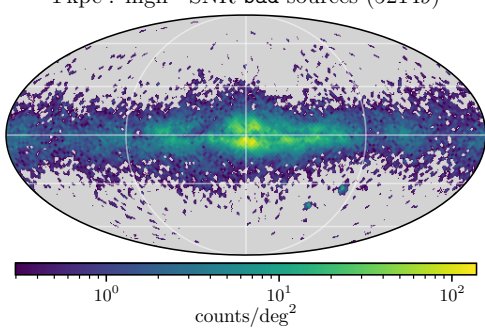

$3 \mathrm{kpc}$ : high-SNR bad sources $(7293)$

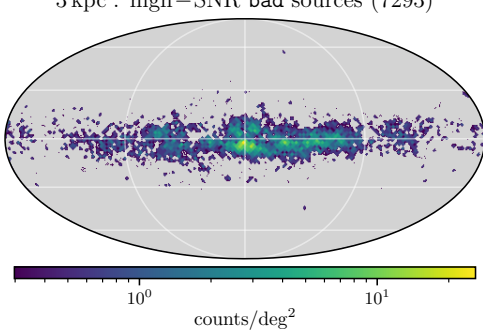

$10 \mathrm{kpc}$ : high-SNR bad sources (393)

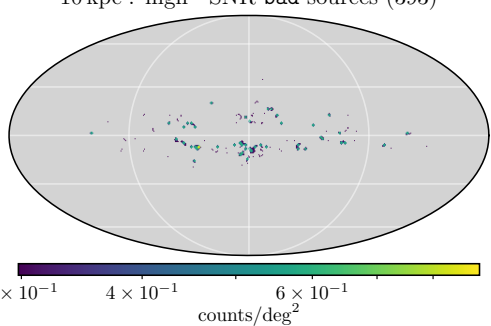

Figure 11. Sky distribution of high-SNR sources in GeDR3mock (left), in eDR3 classified as good (middle), and classified as bad (right column). We show the sky density of sources on a Mollweide projection of Galactic coordinates, with a logarithmic color scale (which is the same for the left and middle panels in each row). From top to bottom, the distance increases logarithmically from $100 \mathrm{pc}$ to $10 \mathrm{kpc}$. We also list the number of sources in each panel, to illustrate the ratio of good to bad sources at the respective measured parallax and the number of expected sources according to GeDR3mock.

\subsubsection{CAMD}

Now we turn at the CAMDs of the high-SNR sources in Fig. 13. The density is shown on a logarithmic color scale. In the $100 \mathrm{pc}$ sample, a prominent difference between eDR3 good sources and GeDR3mock is the lack of WD-MS binaries and sources with photometric excess noise in the latter. However, in GeDR3mock, we see a spreading of the photometry in the brown dwarf regime, which comes from the photometric uncertainties, and which is also visible in the real data. The bad sources have an overdensity at $M_{\mathrm{G}} \approx 15$, which is due to the fact that the number of bad sources increases at smaller parallaxes, as well as the fact that most spurious astrometric solutions are for faint sources. There is also a triangle pointing into the direction of the MS, which may still contain a few good sources. When going further out in distance, the limiting absolute magnitude decreases and more and more extinction sets in. Increasing extinc- 


\section{Jan Rybizki et al.}

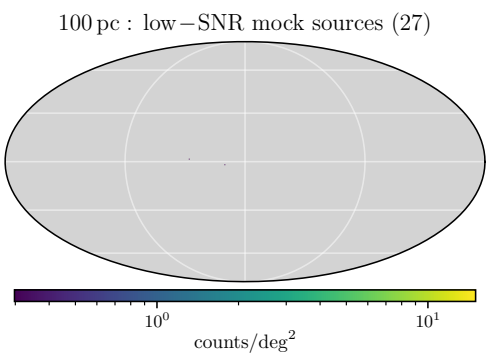

300 pc : low-SNR mock sources (166879)

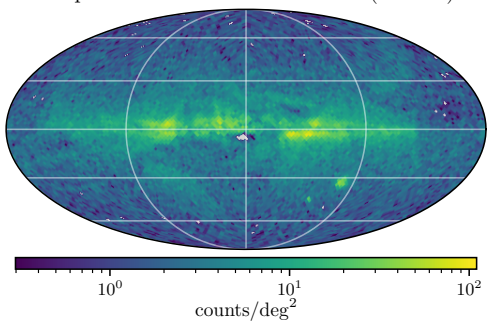

$1 \mathrm{kpc}$ : low-SNR mock sources (1886508)

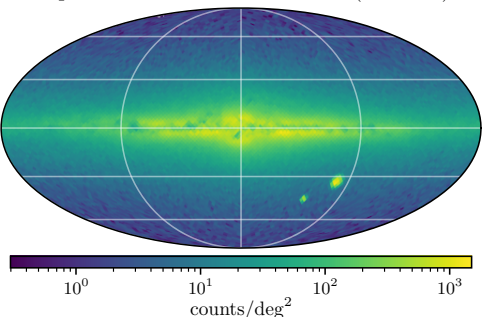

$3 \mathrm{kpc}$ : low-SNR mock sources (1106580)

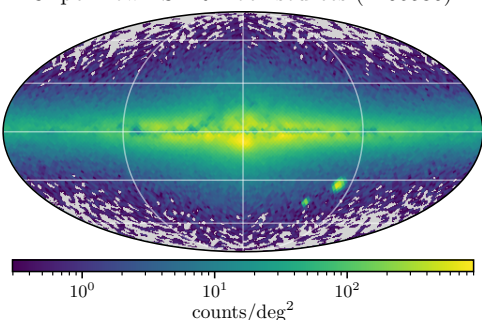

$10 \mathrm{kpc}$ : low-SNR mock sources (1157837)

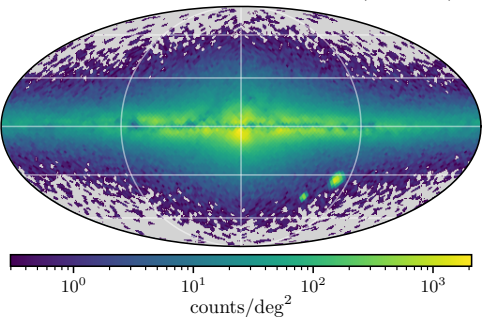

$30 \mathrm{kpc}$ : low-SNR mock sources (1291160)

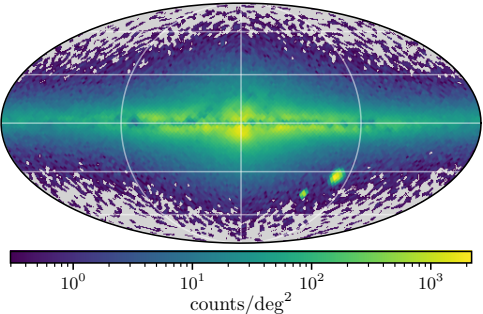

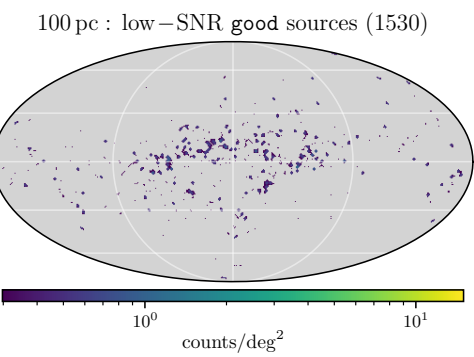

$300 \mathrm{pc}$ : low-SNR good sources (323550)

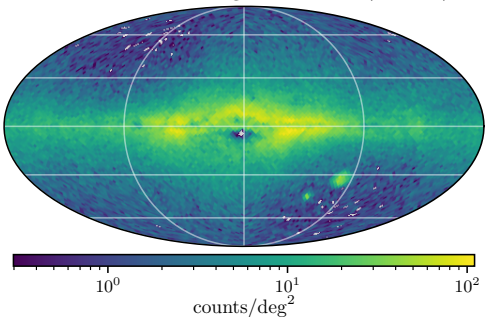

$1 \mathrm{kpc}:$ low-SNR good sources (1605276)

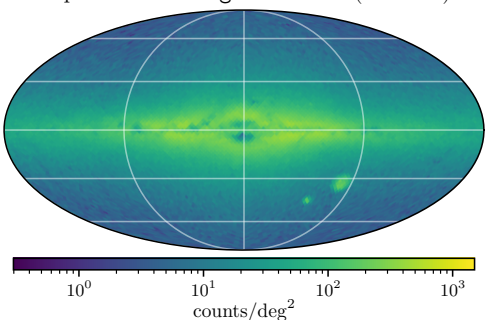

$3 \mathrm{kpc}$ : low-SNR good sources (910865)

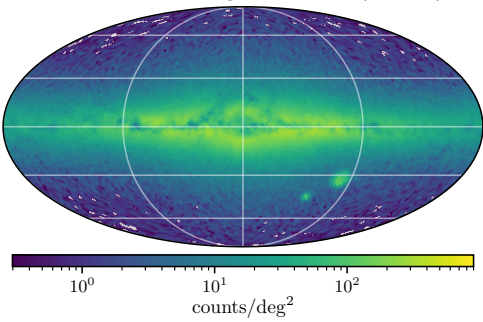

$10 \mathrm{kpc}$ : low-SNR good sources (976972)

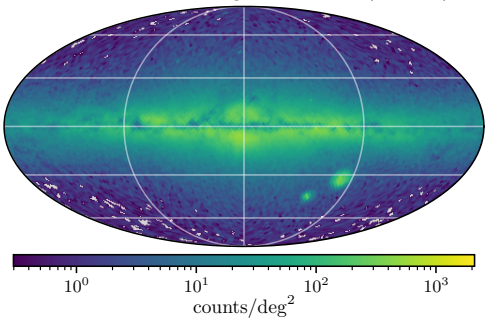

$30 \mathrm{kpc}$ : low-SNR good sources (1140550)

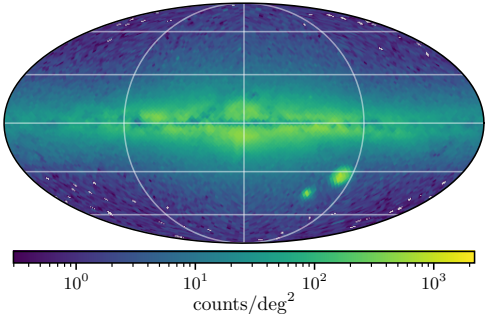

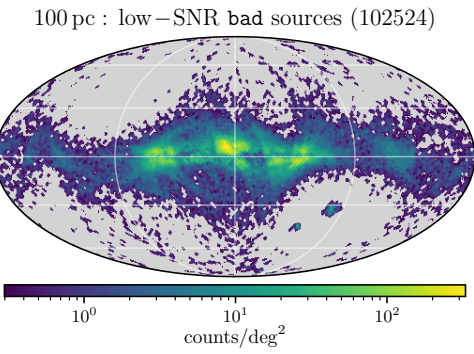

300 pc : low-SNR bad sources (443829)

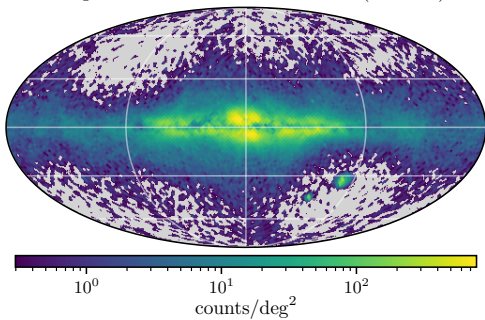

1kpc : low-SNR bad sources (746044)

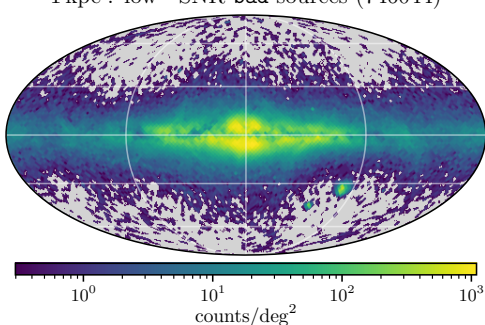

$3 \mathrm{kpc}$ : low-SNR bad sources (185158)

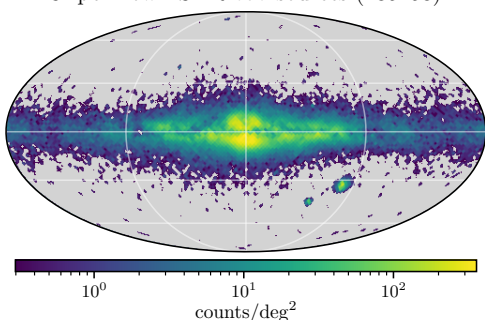

$10 \mathrm{kpc}$ : low-SNR bad sources (200187)

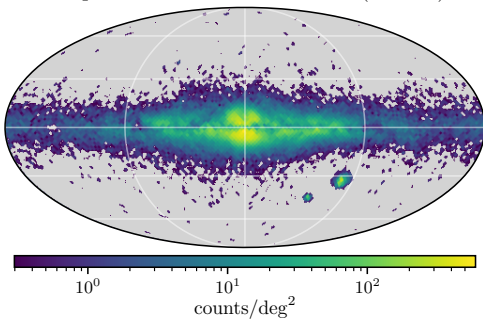

$30 \mathrm{kpc}$ : low-SNR bad sources (285707)

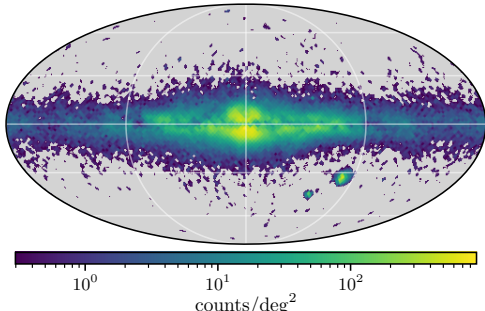

Figure 12. Sky distribution of low-SNR sources in GeDR3mock (left), in eDR3 classified as good (middle), and classified as bad (right column). We show the sky density of sources on a Mollweide projection of Galactic coordinates, with a logarithmic color scale (which is the same for the left and middle panels in each column). From top to bottom, the distance increases logarithmically from $100 \mathrm{pc}$ to $30 \mathrm{kpc}$. We also list the number of sources in each panel, to illustrate the ratio of good to bad sources at the respective measured parallax and the number of expected sources according to GeDR3mock. 
tion is apparent in the elongation of the red clump with increasing distance. At $10 \mathrm{kpc}$, only the brightest stars can be seen in the highSNR sample. In the bad sample, an interesting "fountain" pattern arises at $3 \mathrm{kpc}$, which might be an indication of misclassified red AGB stars.

Looking at the low-SNR CAMDs in Fig. 14, we see that in the first distance bins we expect and see only very faint objects contributing and their $G-R P$ color is smeared out due to the high photometric uncertainty in that regime. Compared to GeDR3mock, the eDR3 data has even larger tails into the red and blue. Otherwise, the good sources closely mimick the GeDR3mock behavior, with a stronger qualitative deviation at $3 \mathrm{kpc}$, where the "fountain" pattern of red AGB stars is visible in GeDR3mock and in the bad sources. This might indicate a misclassification in that regime, as was the case for the high-SNR sources. At $10 \mathrm{kpc}$ in the good sources, we can see massive main sequence stars and turn-off stars, as well as the red clump and perhaps a few sdB stars at the very blue end. For the bad sources, a weak signal of all those populations seems to be present, and again, some red AGB stars might have been wrongly predicted as bad. Overall, most of the physical structure can be seen in the good sample, making us confident that even at low-SNR our astrometric fidelity classifier is useful. Nevertheless, the "fountain" pattern might be a good candidate for further retraining, similar to Section 3.1.2.

\section{CLEANING CAMDS OF SOURCES WITH SPURIOUS COLORS}

We have thus far focused on deriving a purely astrometric fidelity estimator. Here, we generalize this goal somewhat to estimating the reliability of a source's position in the Gaia CAMD.

The fraction of sources with spurious astrometry and/or photometry is likely particularly high in regions of the CAMD that are expected to be sparsely populated astrophysically, such as the region between the main sequence (MS) and WD cooling tracks. Sources in this region of the CAMD are often of particular interest precisely because they are rare. When contaminants with poor astrometry and/or photometry can be efficiently filtered out, sources below the MS and brighter than normal WDs of similar color represent a mix of unresolved WD+MS binaries (Rebassa-Mansergas et al. 2021), cataclysmic variables (e.g. Abrahams et al. 2020), extremely lowmass WDs (e.g. Pelisoli \& Vos 2019), and related transitional binary evolution products (e.g. El-Badry et al. 2021b,a). It is thus desirable to construct a (relatively) clean sample of objects that genuinely fall in this part of color-absolute magnitude space, separating them from normal MS contaminants that scatter into it due to spurious absolute magnitudes and/or colors.

Fig. 15 illustrates how the astrometric fidelity and color contamination parameters can be used to clean such a sample. We begin by selecting from Gaia eDR3 all sources with precise reported parallaxes that fall below the main sequence:

SELECT * from gaiaedr3.gaia_source

WHERE parallax_over_error $>10$ AND

phot_g_mean_mag $+5 * \log 10($ parallax $/ 100)>\left(4 * b p \_r p+\right.$ 2.7)

Here we crudely define the line $M_{G}=4 \times(B P-R P)+2.7$ as the lower boundary of the main sequence. This query yields 526,278 sources, which are shown in the upper left panel of Fig. 15. Almost half of the selected sources fall squarely between the MS and WD cooling track, but we expect that at this stage, a large majority of these sources are spurious. The upper right panel shows the result of applying a cut fidelity_v2 $>0.9$. This reduces the total number of objects by factor of $\sim 2$ and preferentially removes sources farther away from the main sequence, significantly reducing the number of sources likely to be spurious.

We inspected Pan-STARRS 1 images of a random subset of the remaining sources and found that a large majority of them are faint sources that are within $\lesssim 10$ arcsec of a much brighter source. Given that $B P-R P$ colors are calculated from spectra dispersed over a $2 \times 3$ arcsec region (Riello et al. 2021), one expects such sources to have unreliable colors. Their parallaxes may also be less reliable than normal due to centroiding errors, though this has only been found to be severe for sources within $\lesssim 4 \operatorname{arcsec}$ of a brighter source (El-Badry et al. 2021c).

We experimented with a variety of cuts to filter out sources with unreliable colors. One possible approach is to remove all sources with a neighbor that is brighter than the source itself within, say, 5 arcsec (e.g., dist_nearest_neighbor_at_least_Q_brighter $>5$ ). Such a cut would be overly aggressive for neighbors only slightly brighter than the source itself, which are not expected to cause significant photometric contamination at 5 arcsec separation. On the other hand, it would not be aggressive enough for sources with very bright neighbors, which can cause significant photometric contamination even at $>10$ arcsec separations. To account for the farther-reaching contamination from brighter neighbors, we calculate a modified contamination metric as follows.

For each source in Gaia eDR3, we consider all neighboring sources within 30 arcsec as potential contaminants. For each neighbor $i$, we calculate $\Delta G_{i}=G_{\text {source }}-G_{i}$, where $G_{i}$ is the $G$-band magnitude of the neighbors. We then subtract from $\Delta G_{i}$ the angular separation, in arcsec, between the two sources:

$\Delta \tilde{G}_{i}=\frac{\Delta G_{i}}{\operatorname{mag}}-\frac{\theta_{i}}{\operatorname{arcsec}}$.

Finally, we record as norm_dG the largest value of $\Delta \tilde{G}_{i}$ among all neighbors within 30 arcsec. Large values of norm_dG correspond to sources with significant contamination. We find that most sources with norm_dG $<-3$ (e.g, an equally bright neighbor more than 3 arcsec away, or a 2-magnitudes-brighter neighbor more than 5 arcsec away) have unproblematic colors. Sources with no neighbors within 30 arcsec are assigned norm_dG = nan; these are also expected to be uncontaminated. The lower left panel of Fig. 15 shows the effects of applying a cut of norm_dG $<-3$, and the lower right panel shows the sources that are filtered out by it. Most of the removed sources fall between the WD cooling tracks and the MS. There is no reason to expect e.g. cataclysmic variables to preferentially fall close to bright neighbors, so we suspect that these sources are mostly MS stars that have spurious colors or parallaxes.

It is not immediately obvious whether the spurious sources that fall between the MS and WD sequence and are removed by a cut on norm_dG have spurious colors, spurious parallaxes, or both. To distinguish between these possibilities, we investigated the reported parallaxes of sources at high Galactic latitude that (a) are between the MS and WD cooling track, (b) have high astrometric fidelity but are removed by a cut on norm_dG (e.g. bottom panel of Fig. 15), and (c) have a bright companion within 5 arcsec. At high latitudes, most such pairs are gravitationally bound wide binaries (e.g. ElBadry et al. 2021c), and thus the parallaxes of the two stars should be consistent. We found that the reported parallaxes are indeed consistent within $3 \sigma$ in a large majority of pairs, even when one 

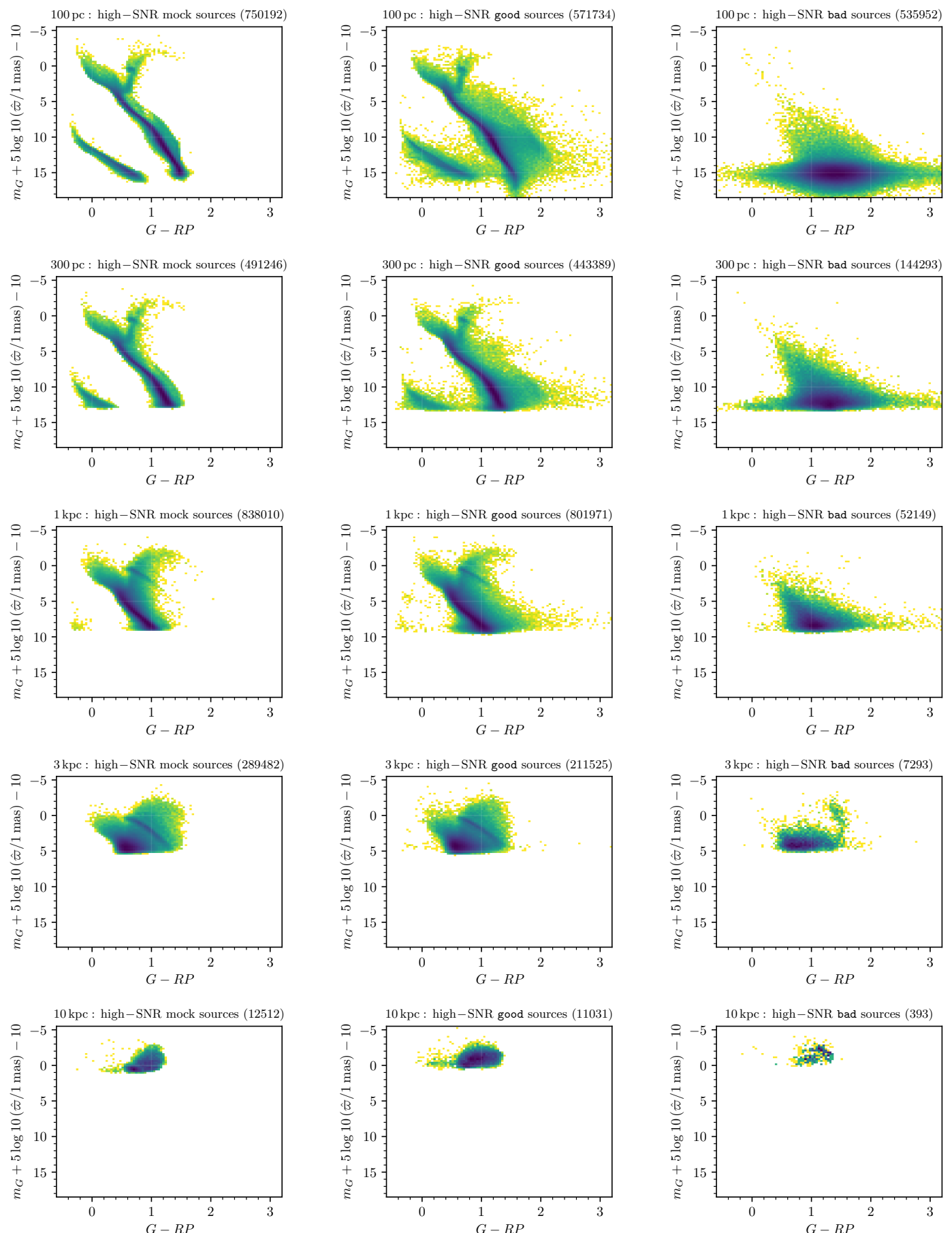

Figure 13. CAMD of high-SNR sources in GeDR3mock (left) in eDR3 classified as good (middle) and classified as bad (right column), on a logarithmic color scale. From top to bottom, the distance increases logarithmically from $100 \mathrm{pc}$ to $10 \mathrm{kpc}$. We also list the number of sources, which illustrates the ratio of good to bad sources at the respective measured parallax, and the number of expected sources according to GeDR3mock.

component falls far from the MS in the Gaia CMD. We conclude that for most sources with high reported astrometric fidelity but spurious CMD position, it is the colors, not the parallaxes, that are incorrect.

In addition to the astrometric fidelity parameter, we report norm_dG values for all sources in Gaia eDR3. We have found that norm_dG is strongly correlated with the phot_bp_rp_excess_factor parameter or the more elaborate photometric excess measure, $C^{*}$ (Riello et al. 2021, eq.6). However, because norm_dG is a geometric quantity, cuts on it are more straightforward to account for in selection function modeling than cuts on phot_bp_rp_excess_factor and other image parameter diagnostics. In addition, norm_dG can be calculated for all Gaia sources, while phot_bp_rp_excess_factor is only reported for sources with measured Gaia colors. We thus recommend filtering on norm_dG as well as astrometric fidelity when a sample with re- 

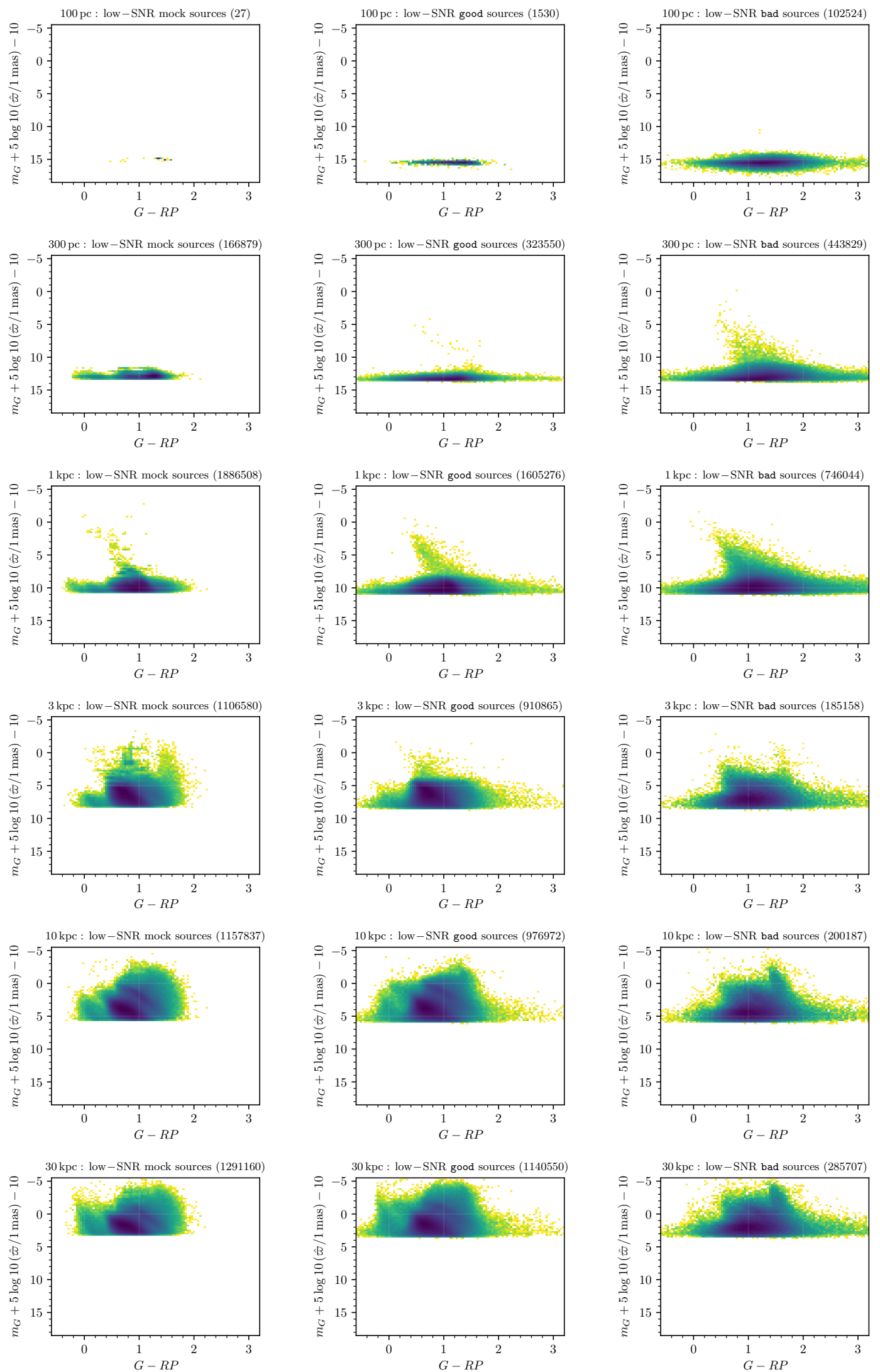

Figure 14. CAMD of low-SNR sources in GeDR3mock (left) in eDR3 classified as good (middle) and classified as bad (right column), on a logarithmic color scale. From top to bottom, the distance increases logarithmically from $100 \mathrm{pc}$ to $30 \mathrm{kpc}$. We also list the number of sources, which illustrates the ratio of good to bad sources at the respective measured parallax, and the number of expected sources according to GeDR3mock. 


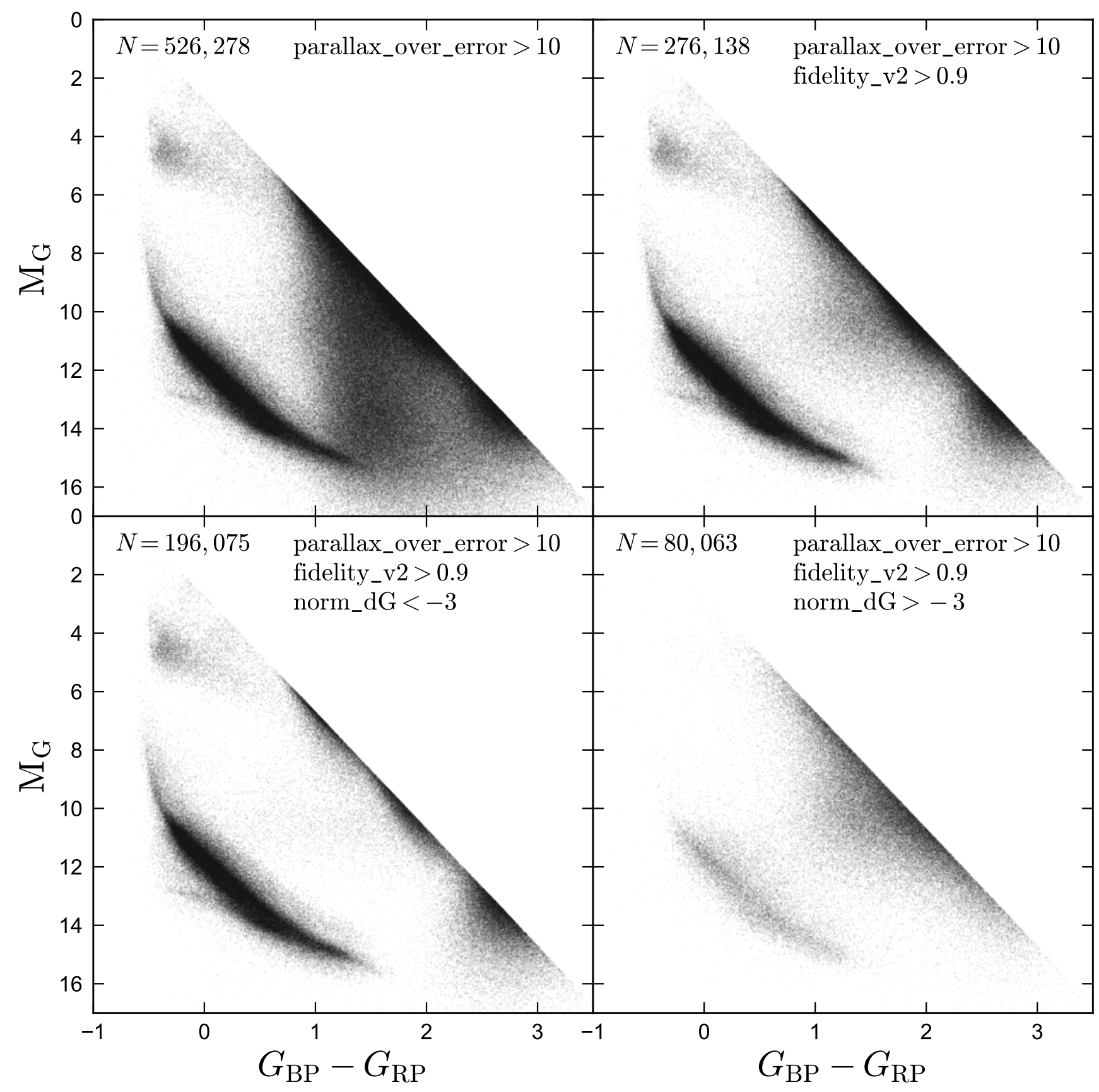

Figure 15. Cleaning of the CAMD using cuts on astrometric fidelity and photometric contamination. Upper left panel shows all sources in Gaia eDR3 with parallax_over_error $>10$ and reported colors and magnitudes that fall well below the main sequence. Upper right panel shows the same sample after sources with fidelity_v2 $<0.9$ have been removed. This eliminates a majority of sources between the main sequence and white dwarf cooling track. Lower left shows the sample after an additional cut of norm_dG $<-3$ (Equation 5), which filters out objects with potentially contaminated photometry. A majority of the surviving sources are on the MS or WD cooling track, and the density of objects between them is significantly reduced. Lower right shows the sources with good astrometric fidelity but potentially contaminated colors; most of these fall between the MS and WD cooling tracks and likely have unreliable colors.

liable colors and parallaxes is required. Cuts on norm_dG will of course remove sources with close neighbors, so cuts on this parameter will preferentially remove objects in wide binaries and dense regions of the sky. Cuts on the astrometric fidelity parameter likely also discriminate against dense regions of the sky, since information about the distance to bright neighbors is used in calculating it.

\section{ACCESS TO THE CATALOG}

Our catalog is hosted at the German Astrophysical Virtual Observatory (GAVO), ${ }^{9}$ in the table gedr3spur.main. ${ }^{10}$ The simplest

\footnotetext{
9 https://dc.zah.uni-heidelberg.de/

10 https://dc.zah. uni-heidelberg.de/tableinfo/gedr3spur. main
} 


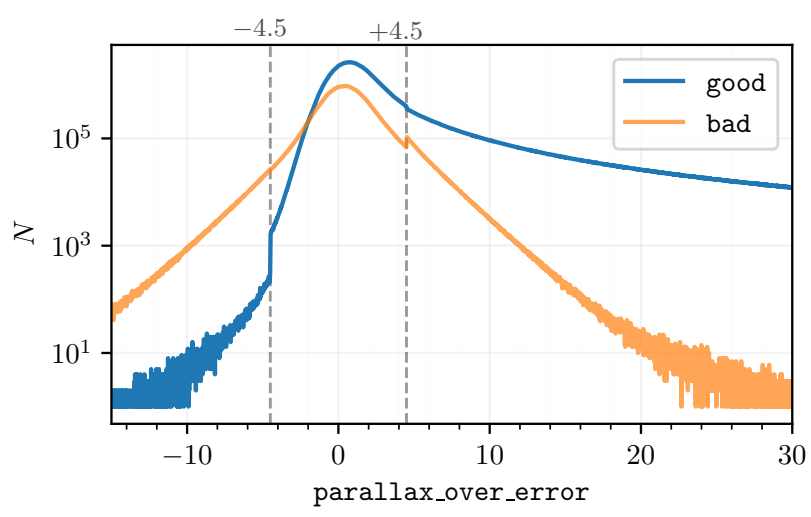

Figure 16. SNR (parallax_over_error) distribution for good and bad sources in Gaia eDR3. The break at $|\mathrm{SNR}|=4.5$, where we switch between the low-SNR and the high-SNR classifier, is shown by dashed gray lines.

way to access the astrometric fidelities for a sample of stars is to crossmatch directly via a Table Access Protocol (TAP) upload join in TOPCAT. ${ }^{11}$ If the local table has Gaia eDR3 source_ids, one can simply query:

\section{SELECT src.*}

FROM gedr3spur.main as src

JOIN TAP_UPLOAD.t1 AS target

-- TAP_UPLOAD.tX needs to be the table number in TOPCAT

USING (source_id)

Because there is a $100 \mathrm{MB}$ upload limit, one can increase the number of sources queried at a time by hiding all columns except source_id. GAVO hosts a light version of Gaia eDR3, containing only the most commonly used columns. One can directly query this light version of Gaia eDR3 and simultaneously cross-match to our astrometric fidelities. For example, the following query returns a histogram of the parallax SNR distribution for the 1,111 million sources in eDR3 classified as good:

SELECT COUNT(*) AS ct,

ROUND (parallax/parallax_error,2) AS bin

FROM gaia.edr3lite -- only contains most important rows

JOIN gedr3spur.main using (source_id)

WHERE fidelity_v2 $>=0.5$ GROUP BY bin

Requiring fidelity_v2 $<0.5$ returns the SNR distribution of the 356 million bad sources. These are depicted in Fig. 16. We see a jump in the number of sources at the $|\mathrm{SNR}|=4.5$ transition between our high- and low-SNR classifier. At the transition point, the number of bad sources increases by $65 \%$, i.e. the high-SNR classifier appears to obtain higher purity. This is unsurprising, as the high-SNR classifier takes |parallax_over_error| into account, which is one of the most informative features. Due to imbalances in the training data, the low-SNR classifier does not take this feature into account (see Section 4.2). Fabricius et al. (2021) estimates the total contamination of the sources with SNR $>5$ to be of the order of $\sim 3$ million (equal to the number of sources with $\mathrm{SNR}<-5$ ). Our classifier finds 11.3 million bad sources in this regime, which constitute $6 \%$ of the 192 million sources with SNR $>5$.

11 The TOPCAT program is described at http://www.star.bris.ac. uk/ mbt/topcat/.
In Fig. 17, we compare v1 with v2 fidelities on all ${ }^{12}$ eDR3 sources with astrometric solutions. We can see that many more sources are classified as good in v2, i.e. 1.1 billion compared to 0.7 billion in $\mathrm{v} 1$. This is presumably due to the new high-density training data.

The upper panels of Fig. 18 show the CAMD of eDR3 sources with SNR $\geq 5$, while the lower panels show the CMD of all eDR3 sources, in three bins of decreasing fidelities, from left to right. For the SNR $\geq 5$ sources in the upper panels, most sources have high fidelities (180 million have fidelity $\geq 2 / 3,10$ million have fidelity $\leq 1 / 3$, and 3 million lie in-between). For all eDR3 sources in the lower panel, we have 1,054 million with fidelity $\geq 2 / 3,304$ million with fidelity $\leq 1 / 3$, and 110 million in between.

The CAMD in the upper left panel shows the richness of Gaia stellar populations visible at high parallax SNR. However, even at lower fidelities (middle and right panel), MS, WD and RC overdensities are visible, together with a fan at very bright and red sources, which might be indicative of good sources that are still misclassified by our model. In the very low fidelity regime (upper right panel), the space between MS and WD has many sources, presumably MS or turn-off stars scattered due to spurious astrometric solutions. It is important to remark, however, that we are only interested in finding spurious astrometric solutions, and not in finding incorrect CAMD positions, which can also result from spurious photometry. One class of sources with spurious photometry is apparent in the upper-left and upper-middle panels for the faint MS, where the $B P-R P$ color is underestimated due to a calibration error (Riello et al. 2021), such that these sources appear too blue. Another class of source with spurious colours arises due to contaminating flux from nearby sources, which can be filtered out using phot_bp_rp_excess_factor or our norm_dG parameter, as described in Section 6.

For the CMDs of all eDR3 sources in the lower panels, we see that bright sources are usually assigned high fidelities (left panel) whereas the lower fidelity sources (middle and right panel) are deficient in bright sources. However, at low fidelities we can see overdensities at the change of window functions from WCOb to WC1 at $m_{G}=13 \mathrm{mag}$ and at $11.5 \mathrm{mag}$ which could correspond to the first gate of WCOb (i.e. gate 12, cf. Fig. 4 of Lindegren et al. 2021a). We also see that low-fidelity sources dominate at the red end.

Finally, in Fig. 19 we show the fraction of bad astrometric solutions, as classified by our v2 model, compared to all astrometric solutions for eDR3. We can see that the densest areas are most vulnerable to spurious solutions, i.e., up to $90 \%$ of sources in the bulge and LMC and can be bad. Dense star clusters in the field also have higher fractions of spurious astrometric solutions. The scanning law is clearly visible as well, and drives up the fraction of bad sources by a few percent.

\section{CONCLUSION}

We have extended the classification of valid and spurious astrometric solutions from the Gaia Catalogue of nearby stars (Gaia Collaboration et al. 2021b) to all 1.47 billion sources in Gaia eDR3 with astrometry. Our training sample of spurious sources is obtained by taking all sources with parallax_over_error $<-4.5$, as well as a set of low-SNR sources identified by the GCNS as bad. The

12 Due to an ingestion error, we missed nearest-neighbor columns of 18 sources. Due to these missing features, these sources did not get assigned final fidelity values. 


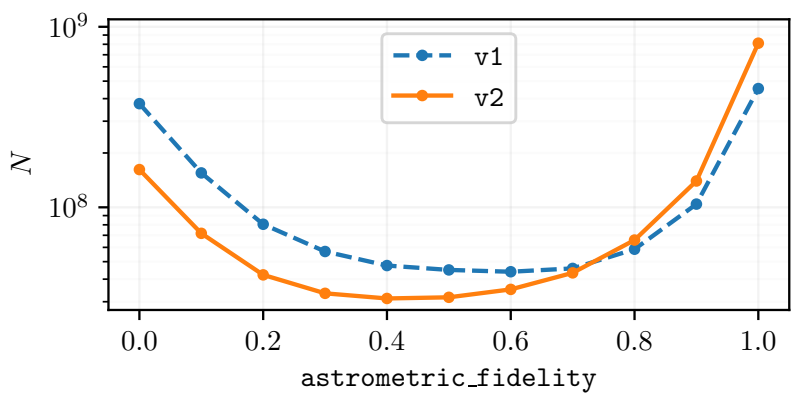

Figure 17. Distribution of astrometric fidelities for eDR3, in bins of 0.1, for both our previous (v1) and our updated (v2) classifier. The v2 classifier marks fewer sources as bad, particularly in dense regions of the sky, due to our addition of good training datasets covering this regime. We expect our v2 classifier to perform better (in particular, to achieve higher completeness) in dense regions of the sky.

bulk of our training sample of good astrometric solutions is obtained by taking all sources with a PS1 crossmatch in parts of the sky where no sources with parallax_over_error $<-3.6$ exist. We additionally identify good sources that lie on the main sequence on a PS1 CAMD, as well as sources in globular clusters with measured parallaxes consistent with the cluster parallax (Vasiliev \& Baumgardt 2021).

We train two neural network models: one for high-parallaxSNR and one for the low-parallax-SNR regime (divided at $|\mathrm{SNR}|=$ 4.5), which take astrometric quality parameters from eDR3 and information about distances to nearby sources as inputs.

Our validation shows that we outperform simple cuts but also logistic models that take into account a linear combination of our features as well as ExtraTrees. Across a wide range of parallaxes, sources that our classifier identifies as good are more plausibly distributed both on the sky and on a CAMD than sources that our classifier identifies as bad. Similarly, the spatial distribution (based on measured parallaxes) of OBA stars in the Milky Way midplane that our classifier marks as having good astrometric solutions is plausible, while the spatial distribution of stars with bad astrometric solutions is highly unphysical. The parallaxes of sources that our classifier identifies as good in the LMC and globular/open clusters are normally distributed with respect to their host parallaxes, with smaller scatter than sources that our classifier identifies as bad. Sources classified as good also have significantly lower $\chi^{2}$ when comparing to OGLE proper motions. Sources classified as bad usually occur in high-density regions, e.g. in the bulge and disc region and in the Magellanic clouds.

The astrometric fidelities presented in this paper can be used in place of simpler cuts on Gaia catalog columns, such as ruwe and astrometric_excess_noise, to filter out sources with spurious astrometric solutions. In most regimes, astrometric fidelities should yield a purer and more complete sample of sources with reliable astrometric solutions. In addition to the astrometric fidelities, we also provide simple diagnostics of the level of photometric contamination from bright neighbors (Section 6), which can be used to filter out sources with unreliable colors.

We provide the community with all the data and an online python notebook that can rerun the whole training and validation procedure automatically. This allows for retraining with additional sources that the user identified as good or bad, e.g. from (in-)compatible photometry and astrometry of dwarf galaxy sources. Worthwhile improvements could also come from training special purpose models, e.g., for dense regions, binaries or for sources that have all photometric bands available (i.e. BP, RP which would allow for additional features like phot_bp_rp_excess_factor). One could also re-run for GDR2 data and for future releases. Two more improvements that come to mind are taking the parallax zero point and parallax error scalings into account. Creating a selection function for the good sources would be a worthwhile project on its own.

\section{ACKNOWLEDGEMENTS}

We thank the anonymous referee for swift, constructive and thorough feedback on the manuscript. It is a pleasure to thank Claus Fabricius, Jordi Portell for help in understanding the astrometric solution. We thank Anthony Brown, Eugene Vasiliev, Zephyr Penoyre, Lennart Lindegren, Andy Everall, Adam Riess and Ron Drimmel for valuable feedback at the EDR3 workshop. We are also thankful for input and discussions with the MPIA Gaia and MW group. Furthermore, the authors would like to thank Douglas P. Finkbeiner and Joshua S. Speagle for helpful discussions and suggestions.

This work has made use of data from the European Space Agency (ESA) mission Gaia, processed by the Gaia Data Processing and Analysis Consortium (DPAC). Funding for the DPAC has been provided by national institutions, in particular the institutions participating in the Gaia Multilateral Agreement.

This research or product makes use of public auxiliary data provided by ESA/Gaia/DPAC as obtained from the publicly accessible ESA Gaia SFTP.

This work was funded by the DLR (German space agency) via grant 50 QG 1403 .

GG acknowledges funding from the Alexander von Humboldt Foundation, through the Sofja Kovalevskaja Award.

The OGLE project has received funding from the National Science Centre, Poland, grant MAESTRO 2014/14/A/ST9/00121 to AU.

JR will not travel anywhere by aeroplane for the purpose of promoting this paper.

Software: TOPCAT (Taylor 2005), Tensorflow (Abadi et al. 2016), Keras (Chollet et al. 2015), HEALpix (Górski et al. 2005), astropy (The Astropy Collaboration et al. 2018), matplotlib (Hunter 2007).

\section{Data availability}

The data underlying this article are available in the article and in its online supplementary material.

\section{REFERENCES}

Abadi M., et al., 2016, TensorFlow: Large-Scale Machine Learning on Heterogeneous Distributed Systems (arXiv: 1603.04467)

Abrahams E. S., Bloom J. S., Mowlavi N., Szkody P., Rix H.-W., Ventura J.-P., Brink T. G., Filippenko A. V., 2020, arXiv e-prints, p. arXiv:2011.12253

Anders F., et al., 2019, A\&A, 628, A94

Bailer-Jones C. A. L., 2015, PASP, 127, 994

Bailer-Jones C. A. L., Rybizki J., Fouesneau M., Mantelet G., Andrae R., 2018, AJ, 156, 58

Chambers K. C., et al., 2016, arXiv e-prints, p. arXiv:1612.05560

Chollet F., et al., 2015, Keras, https://keras.io 

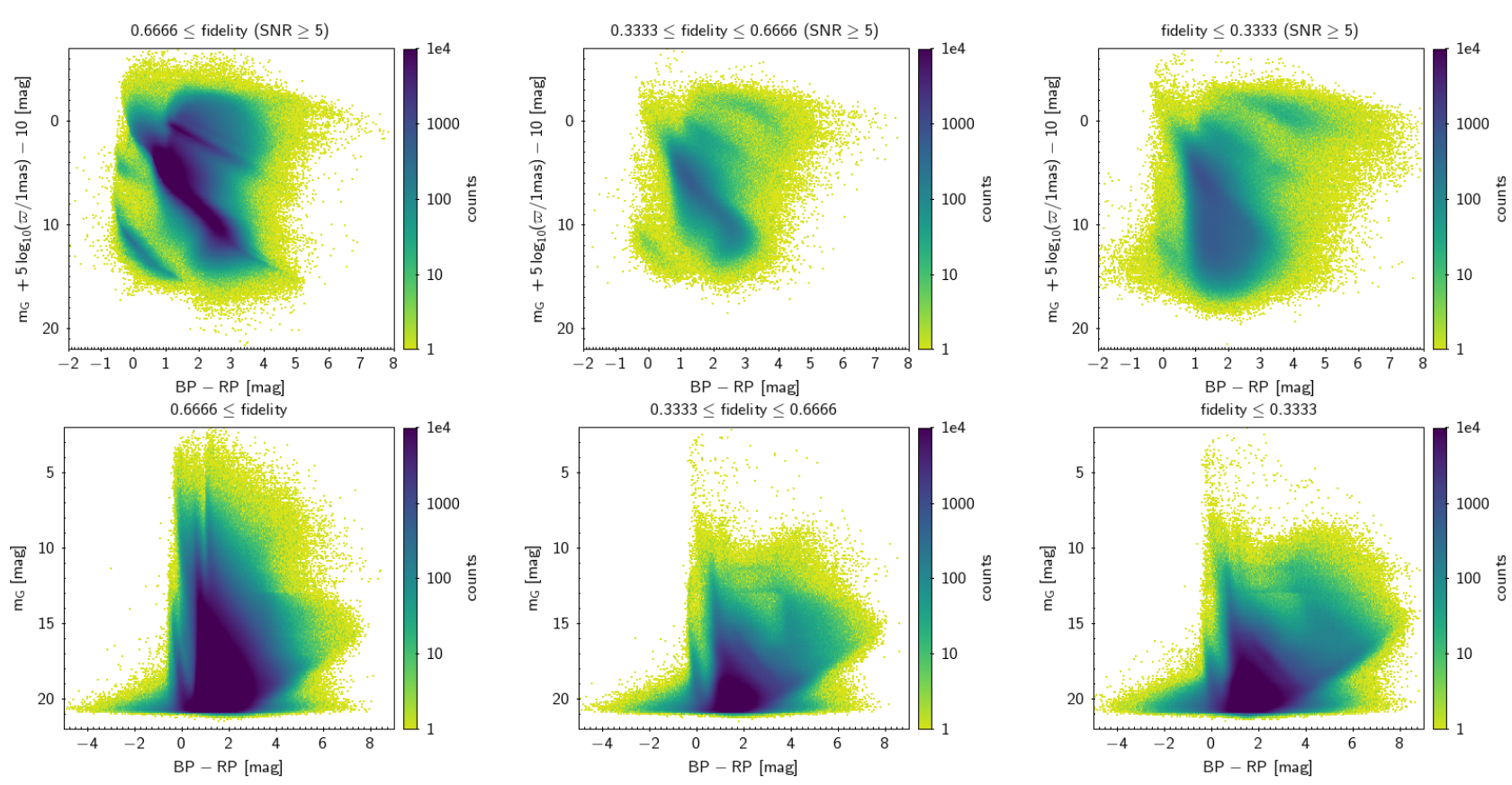

Figure 18. Upper panels: CAMD of SNR $\geq 5$ sources split into fidelity_v2 bins, decreasing from left to right. All three panels share the same color bar. Lower panels: as upper panels, but for CMD and all sources.

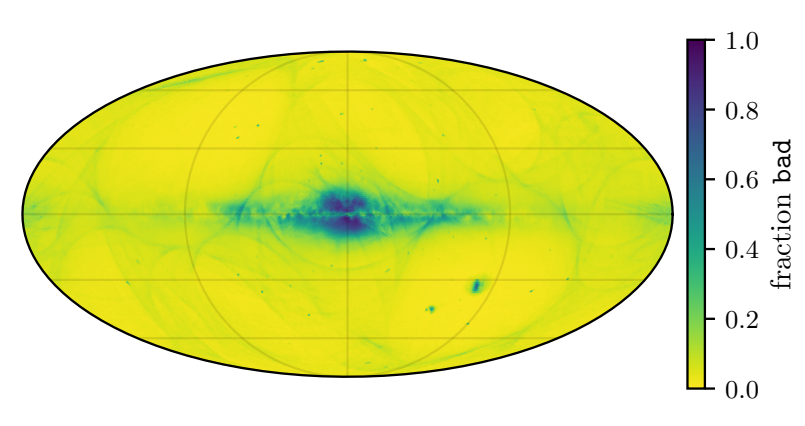

Figure 19. Fractions of bad sources with respect to all eDR3 sources with astrometric solutions over the sky, using a Mollweide projection of Galactic coordinates.

El-Badry K., Rix H.-W., Quataert E., Kupfer T., Shen K. J., 2021a, arXiv e-prints, p. arXiv:2108.04255

El-Badry K., et al., 2021b, MNRAS, 505, 2051

El-Badry K., Rix H.-W., Heintz T. M., 2021c, MNRAS, 506, 2269

Fabricius C., et al., 2021, A\&A, 649, A5

Gaia Collaboration et al., 2018, A\&A, 616, A10

Gaia Collaboration et al., 2021a, A\&A, 649, A1

Gaia Collaboration et al., 2021b, A\&A, 649, A6

Gentile Fusillo N. P., et al., 2021, arXiv e-prints, p. arXiv:2106.07669

Geurts P., Ernst D., Wehenkel L., 2006, Mach. Learn., 63, 3-42

Goodfellow I., Bengio Y., Courville A., 2016, Deep Learning. MIT Press, http://www . deeplearningbook.org

Górski K. M., Hivon E., Banday A. J., Wandelt B. D., Hansen F. K., Reinecke M., Bartelmann M., 2005, ApJ, 622, 759

Green G., 2018, The Journal of Open Source Software, 3, 695

Green G. M., Schlafly E., Zucker C., Speagle J. S., Finkbeiner D., 2019, ApJ, 887, 93

Hunter J. D., 2007, Computing in Science \& Engineering, 9, 90

Kingma D. P., Ba J., 2014, arXiv e-prints, p. arXiv: 1412.6980

Lindegren L., et al., 2021a, A\&A, 649, A2

Lindegren L., et al., 2021b, A\&A, 649, A4
Luri X., et al., 2018, A\&A, 616, A9

Pelisoli I., Vos J., 2019, MNRAS, 488, 2892

Rebassa-Mansergas A., et al., 2021, MNRAS, 506, 5201

Riello M., et al., 2021, A\&A, 649, A3

Rybizki J., et al., 2020, PASP, 132, 074501

Schlegel D. J., Finkbeiner D. P., Davis M., 1998, ApJ, 500, 525

Taylor M. B., 2005, in Shopbell P., Britton M., Ebert R., eds, Astronomical Society of the Pacific Conference Series Vol. 347, Astronomical Data Analysis Software and Systems XIV. p. 29

The Astropy Collaboration et al., 2018, AJ, 156, 123

Torra F., et al., 2021, A\&A, 649, A10

Udalski A., Szymański M. K., Szymański G., 2015, Acta Astron., 65, 1

Vasiliev E., Baumgardt H., 2021, MNRAS, 505, 5978

Zari E., Rix H. W., Frankel N., Xiang M., Poggio E., Drimmel R., Tkachenko A., 2021, A\&A, 650, A112

Zinn J. C., 2021, AJ, 161, 214

\section{APPENDIX A: FEATURES}

In order to provide a bit more insight into the features used, Fig. A1 shows their correlation matrix in the training dataset.

Below, we also show the ExtraTree importance of each feature (averaged over 3 random determinations and normalised to the most important). These importance estimates do not necessarily carry over to the neural network model, and are only shown in order to give a rough idea of the discriminatory power of each feature: 1.000 astrometric_excess_noise

0.743 dist_nearest_neighbor_at_least_0_brighter

0.367 parallax_over_error

0.339 ipd_frac_multi_peak

0.323 astrometric_sigma5d_max

0.274 ruwe

0.250 astrometric_params_solved

0.213 astrometric_gof_al

0.200 visibility_periods_used

0.139 parallax_error 

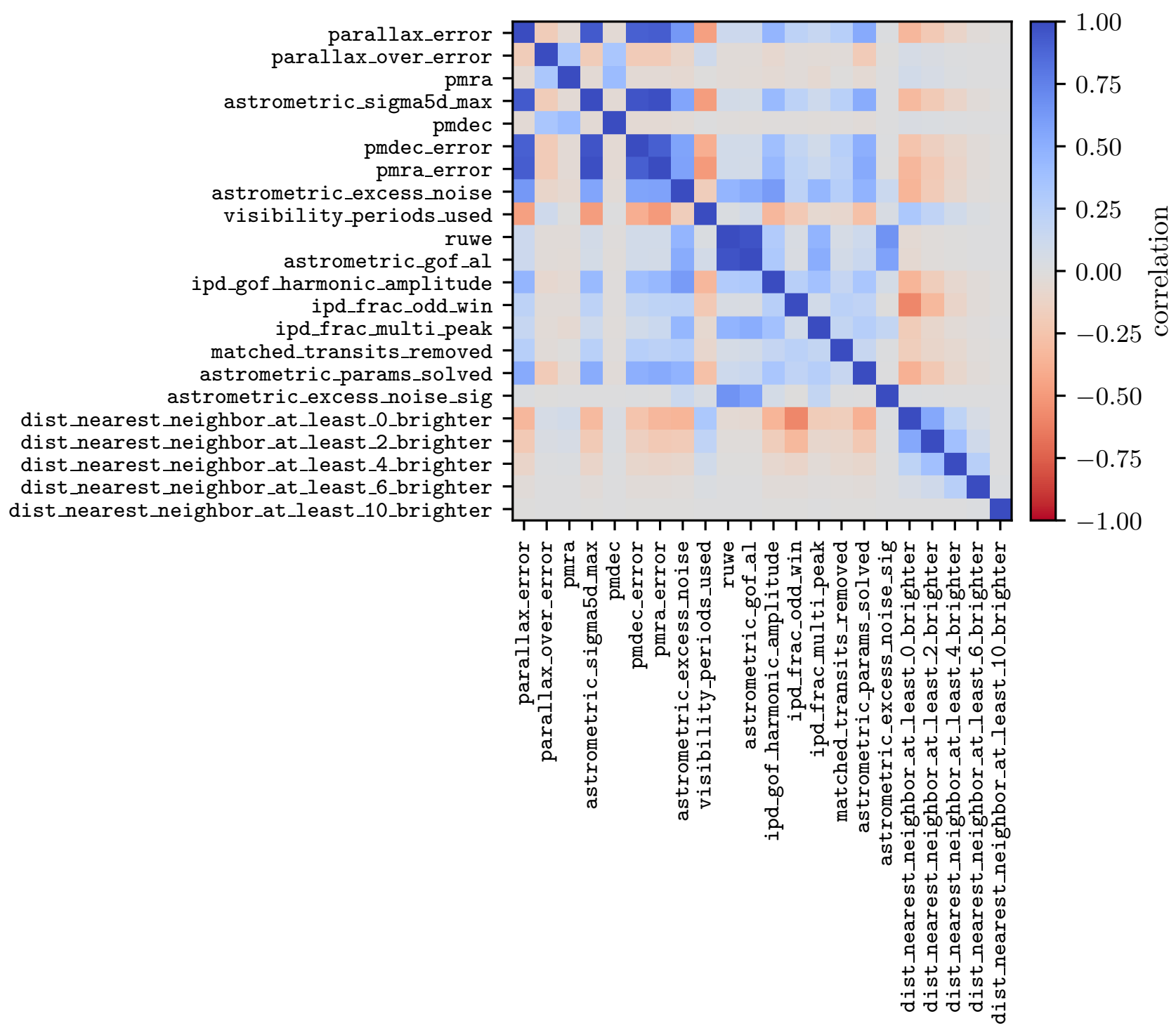

Figure A1. Spearman's cross correlation matrix of the features in the training dataset. Positive correlations are shown in blue and anti-correlations in red. Gray indicates no correlation.

0.136 ipd_gof_harmonic_amplitude

0.082 pmdec_error

0.060 pmra_error

0.060 astrometric_excess_noise_sig

$0.048 \mathrm{pmra}$

0.048 pmdec

0.038 dist_nearest_neighbor_at_least_2_brighter

0.020 ipd_frac_odd_win

0.007 matched_transits_removed

0.002 dist_nearest_neighbor_at_least_4_brighter

0.000 dist_nearest_neighbor_at_least_6_brighter

0.000 dist_nearest_neighbor_at_least_10_brighter

\section{APPENDIX B：GEDR3MOCK}

In order to compare the distance slices with expected distributions in the CMD and sky we query the GeDR3mock (Rybizki et al. 2020) using the following query:

\footnotetext{
SELECT g. *

FROM

SELECT source_id,

parallax_obs/parallax_error_scaled as parallax_over_error,

GAVO_NORMAL_RANDOM( phot_g_mean_mag,

phot_g_mean_mag_error) AS phot_g_mean_mag, GAVO_NORMAL_RANDOM( phot_rp_mean_mag,

phot_rp_mean_mag_error) AS phot_rp_mean_mag, GAVO_NORMAL_RANDOM( phot_bp_mean_mag,

phot_bp_mean_mag_error) AS phot_bp_mean_mag,

-- Using pseudo-observed instead of true photometry parallax_error_scaled, parallax_obs as parallax FROM (
} 
SELECT y.*,

GAVO_NORMAL_RANDOM( parallax,

parallax_error_scaled) AS parallax_obs

-- Pseudo-observed parallaxes by sampling the error FROM (

SELECT $z$.*,

POWER (10, ((LOG (parallax_error)/LOG $(10)+1) * 1.3)-1)$ AS

parallax_error_scaled

-- Rescaling the error according to the GCNS paper FROM gedr3mock. main as $z$

-- WHERE source_id BETWEEN 6001046503471185920 AND 6001609453424607231

-- uncomment above line in order to run with synchronous mode otherwise takes 1-2 hours

$$
\begin{aligned}
& \text { ) as } y \\
& \text { as } \mathrm{x}
\end{aligned}
$$

WHERE

(x.parallax_obs $>8$ ) OR

(x.parallax_obs $>1$

AND $x$. parallax_obs $<1.01)$ OR

(x.parallax_obs $>3.3$

AND $x$. parallax_obs $<3.4)$ OR

(x.parallax_obs $>0.1$

AND $x$.parallax_obs $<0.101)$ OR

(x.parallax_obs $>0.333$

AND $x$.parallax_obs $<0.334)$ OR

(x.parallax_obs $>0.0325$

AND $x$.parallax_obs $<0.034)$

-- specifying the different parallax slices from our validation set

) as $\mathrm{g}$

JOIN gens.maglims6 AS lim

ON (g. source_id/140737488355328=lim.hpx)

WHERE phot_g_mean_mag<lim.magnitude_90

-- dropping sources in gedr3mock that are below Gaia's magnitude limit

This yields pseudo-observed quantities as the photometry and parallaxes are sampled with their respective uncertainties. We also apply the GeDR3 G magnitude limits per level 6 HEALpix which we approximate with the 90th percentile of the GeDR3 G magnitude distribution per HEALpix for sources which have a parallax measurement (Gaia Collaboration et al. 2021b).

This paper has been typeset from a $\mathrm{TEX}_{\mathrm{X}} / \mathrm{L} \mathrm{T} \mathrm{EX}$ file prepared by the author. 